\title{
Lexis
}

Journal in English Lexicology

2| 2008

Lexical Submorphemics

\section{Les 'mots en bl-' du lexique anglais : étude de la structuration des données dans une optique lexico-cognitive}

\section{Line Argoud}

\section{OpenEdition}

Journals

Édition électronique

URL : http://journals.openedition.org/lexis/698

DOI : $10.4000 /$ lexis.698

ISSN : 1951-6215

Éditeur

Université Jean Moulin - Lyon 3

Référence électronique

Line Argoud, "Les 'mots en bl-' du lexique anglais : étude de la structuration des données dans une optique lexico-cognitive», Lexis [En ligne], 2 | 2008, mis en ligne le 10 novembre 2008, consulté le 01 mai 2019. URL : http://journals.openedition.org/lexis/698; DOI : 10.4000/lexis.698

\section{(C) $\Theta \Theta \Theta$}

Lexis is licensed under a Creative Commons Attribution-NonCommercial-NoDerivatives 4.0 International License. 


\title{
Les 'mots en $b l$-' du lexique anglais : étude de la structuration des données dans une optique lexico-cognitive
}

Line Argoud ${ }^{8}$

\section{Résumé}

Cette étude se donne pour corpus l'ensemble des mots du lexique anglais présentant le groupe consonantique $b l$ - à l'initiale. Notre objectif global est, dans un premier temps, de tenter de montrer que l'invariance phonologique de cette classe heuristiquement constituée de 'mots en $b l$-' véhicule une invariance notionnelle en profondeur: les sens de deux sous-classes importantes de 'mots en $b l$-' permettent 1 'accès à des représentations mentales dont nous postulons que le soubassement repose sur le 'schème mental' $<$ CONTAINER $>$ (Lakoff \& Johnson). Dans un second volet, nous proposons d'étudier le rôle joué par l'aspectualité lexicale dans nos données, à partir des traits définitoires des 'mots en bl-' tels qu'ils apparaissent dans le Oxford English Dictionary et le English Dialect Dictionary.

Mots-clés : aktionsart - aspectualité - cognition - corps humain - invariance submorphémique - motivation

$* * *$

\begin{abstract}
In this paper, I explore the class of English lexical items beginning with $b l$-. My objective is twofold: firstly, I present the hypothesis that the phonological invariance of this heuristicallyformed class corresponds to a notional invariance within two important subsets of ' $b l$ - words'. I claim that this notional invariance may be traced back to mental representations whose underlying source is the $<$ CONTAINER $>$ schema (Lakoff \& Johnson). Secondly, I consider the part played by aspect in the data provided by the entries for ' $b l$ - words' in two dictionaries, the Oxford English Dictionary and the English Dialect Dictionary.
\end{abstract}

Keywords: aktionsart - aspect - cognition - human body - submorphemic invariance - motivation

\footnotetext{
${ }^{8}$ Doctorante agrégée, Grenoble - Université de Toulouse-Le Mirail.
} 


\section{Préambule : démarche et considérations théoriques}

Cette étude se donne pour objet la classe des 'mots en $b l$-', classe formée selon des critères heuristiques: ces mots présentent une invariance phonologique, celle du groupe consonantique $b l$ - à l'initiale du mot. Nous en examinons ici deux sous-classes, celle des 'mots en $b l$-' dont les sens renvoient à la notion que nous nommerons $<$ Flot / Courant $>$, et celle dont les sens renvoient à la notion $<$ Vision / Luminosité $>$. Le segment $b l$-, appelé parfois 'phonesthème' (Firth 1930), dont le statut, avec d'autres groupes consonantiques initiaux, est l'objet de controverses au sein de la communauté des linguistes, est envisagé ici comme un submorphème, dont la définition nous est fournie par Crystal : " A submorpheme is a term sometimes used to refer to a part of a morpheme that has recurrent form and meaning, such as the sl- beginning of slimy, slug, etc. » (Crystal 2003 : 301).

Notre analyse comprend plusieurs dimensions : lexicologique, sémantique, étymologique, et mythique. Au terme de ces quatre directions de recherche, que nous évoquerons ici brièvement, nous tenterons d'expliquer les phénomènes observés à la lumière d'une approche cognitive. Nous chercherons en particulier à cerner quels liens peuvent entretenir, au niveau cognitif, les deux notions considérées; et quel rôle semble jouer le schéma corporel à ce sujet. Nous explorerons l'hypothèse selon laquelle $b l$ - serait la trace en surface d'une invariance submorphémique permettant l'accès à des représentations dans lesquelles le schème fondamental <CONTAINER>, appliqué au corps humain, paraît jouer le rôle de soubassement initial. L'invariance postulée est d'ordre notionnel plutôt que sémantique, car force est de constater que tous les membres des deux sous-classes de 'mots en $b l$-' étudiées ne partagent pas à proprement parler une communauté de sens, mais que ces sens renvoient à des notions précises qu'il nous appartiendra d'appréhender.

L'analyse sub-lexicale des 'mots en $b l$-' s'apparente, sur le plan méthodologique, à celle des 'mots en th-' ou en ' $w h$-' de la grammaire anglaise, à ceci près qu'il ne s'agit pas là de l'analyse de sous-ensembles, mais d'ensembles. Il y a, en effet, analogie entre notre proposition d'envisager le groupe consonantique initial $b l$ - dans le cadre lexical, et celle de concevoir wh- ou th- comme "la codification d'une même activité mentale » en grammaire (Lapaire \& Rotgé 1998: 608). Certaines grammaires, qui s'inscrivent dans le sillage de la théorie des opérations énonciatives de Culioli, attribuent à "chacun des marqueurs WH- ou TH- », dans la classe des mots grammaticaux en WH- et en TH- respectivement, une « valeur propre que l'on retrouve dans chacune des combinaisons dans lesquelles il apparait » (ibid. : 608). Tout comme, en grammaire, l'objectif est, à partir des 'valeurs d'emplois' ou 'effets de sens' en discours, " de tenter de dégager, en langue, une valeur centrale constante, compatible avec toutes ces utilisations » (ibid. : 40), il s'agira pour nous, à partir des différents sens pris par les formes lexicales attestées dans les dictionnaires consultés, de cerner au plus près les domaines notionnels auxquels renvoient ces sens. La différence réside en ce que notre lieu d'observation n'est pas « le foisonnement sans cesse renouvelé du discours » (ibid. : 40), mais les manifestations sémantiques attestées par le lexicographe.

Nous ne consacrerons pas une section spéciale à l'exposé de notre cadre théorique, mais évoquerons ce dernier au fil de cette étude, afin de définir certains termes qui doivent s'entendre comme éléments d'une nomenclature spécifique renvoyant à une théorie particulière. Tel est le cas, par exemple, de la «notion» culiolienne, de la projection conceptuelle métaphorique proposée par Lakoff \& Johnson (2003), ou des schèmes mentaux (image schemas) de Johnson (1987). Globalement, l'esquisse du modèle cognitif que nous proposons s'appuie sur les travaux de Culioli (1990), de Sweetser (1990), et de Gärdenfors 
(2000). En ce qui concerne l'approche cognitive, la Conceptual Metaphor Theory (CMT) élaborée par Lakoff \& Johnson demeure notre référence prépondérante, ainsi que certaines de ses élaborations, comme les réseaux conceptuels métaphoriques. Nous nous appuierons également sur la notion de schèmes mentaux (Johnson 1987, Lakoff 1987, Lakoff \& Johnson 1999 et 2003), afin de formuler des hypothèses quant aux liens qu'entretiennent, selon nous, les deux domaines notionnels $<$ Vision / Luminosité $>$ et $<$ Courant / Flot $>$.

Nous examinerons également l'ensemble des données en invoquant un certain type de conceptualisation d'événements, que Talmy nomme «Fictive Motion » (Talmy 2000). Enfin, nous emprunterons à Langacker l'un de ses concepts fondamentaux, celui des modèles en réseau (network model), touchant à l'organisation des catégories, pour rendre compte de la polysémie de blood; et utiliserons la notion d' "Active Zone » (Langacker 1987, 2002) afin de mieux cerner les parties du corps qui interviennent de façon systématique dans les procès désignés par certains 'verbes en $b l$-'.

Nous avons utilisé deux dictionnaires : l'OED ${ }^{9}$ pour l'anglais contemporain, et $1^{\prime} E D D^{10}$ pour l'anglais dialectal de la fin du XIXe siècle. A partir des bases lexicales des noms, des verbes, et des adjectifs, nous avons établi dans nos tableaux préliminaires 174 entrées pour l'OED, et 264 entrées pour l'EDD. La plupart de ces lexèmes sont communs aux deux dictionnaires (bladder, blow); d'autres sont des formes dialectales, souvent obsolètes, et attestées uniquement dans l'EDD, comme blandigo "shower of rain », ou blawp « to belch, to heave up water ». Enfin, certains, plus rares, n'apparaissent que dans l'OED, tels que blivit "US slang: something useless ». Notre but étant de nous concentrer sur les bases lexicales, nous avons éliminé : les mots formés par composition, extrêmement nombreux en ce qui concerne les formes désignant des couleurs; les mots formés par dérivation ${ }^{11}$ : comme, par exemple, blazer $(<$ blaze + er $)$; les mots dont les traits définitoires indiquent explicitement un renvoi à un autre mot; les mots qui ne représentent qu'une variante de prononciation ; les mots qui ne font partie de la classe des 'mots en $b l$-' que par le résultat d'un processus de syncope (bliv $=$ «to believe [pron. of believe] »); les interjections; les formes résultant d'emprunts récents à une autre langue ; et, enfin, les mots dérivés d'un nom propre (bloomer).

\section{Analyse sémantique}

\subsection{Sens les plus fréquents}

\subsubsection{Pour les noms}

- Un courant, un flux (blast). La substance déplacée ne se limite pas à l'air, mais peut être un liquide, voire un mélange d'air, d'eau, et de substances solides. Dans le cas de phénomènes météorologiques, comme des rafales ou des précipitations, il est souvent difficile de distinguer l'action du vent de celle de la pluie ou de la neige ;

- Un renflement qui peut s'apparenter à une bulle, c'est-à-dire correspondant souvent à l'action ou le résultat de ce déplacement d'air ou de liquide : blain, blister, bladder, blob et de

\footnotetext{
${ }^{9}$ The Oxford English Dictionary, 2 ème éd., 1989.

${ }^{10}$ The English Dialect Dictionary (Wright 1898-1905).

${ }^{11}$ Comme en ce qui concerne la couleur (mots formés à partir de black et de blue), les mots formés par composition ou / et dérivation à partir de blood sont également extrêmement nombreux : nous n'en comptons pas moins de sept pages et soixante entrées dans l'OED.
} 
nombreuses formes dialectales attestées dans l'EDD. L'action de la bouche semble à l'origine déterminante, l'air étant expulsé comme pour former intentionnellement une vésicule, mais le domaine des éléments naturels est également bien représenté (blubber) ;

- Un objet ou une partie d'objet qui attire le regard, souvent par sa luminosité (blaze), sa couleur claire ou bariolée (blaze " white mark on a horse's face »). Cette notion est beaucoup plus fréquemment actualisée par des adjectifs (voir ci-dessous).

\subsubsection{Pour les verbes}

- L'action de produire un courant d'air, ou d'injecter de l'air dans un objet. Si l'origine de ce flux est dans la plupart des cas la bouche (blow), l'air ou le liquide peuvent s'écouler d'autres parties du corps (air ou mucus chassés par le nez (blast), larmes déversées par les yeux $($ blirt )). Enfin, il s'agit fréquemment de phénomènes météorologiques, extérieurs au corps, où l'agent qui donne naissance au courant est le vent, ou la mer (bluster). L'on trouve souvent des formes polysémiques dont les sens renvoient au domaine du corps et à celui des éléments naturels (blast) ;

- L'émission par la bouche de divers sons ou bruits, pouvant s'apparenter ou non à un 'flot de paroles'. C'est en principe de la bouche humaine que sortent ces cris et éclats de voix (blab, blether), mais les animaux ne sont pas en reste (bleat);

- Certains mouvements des yeux (blink) ou diverses actions faisant intervenir la vision : soit l'activité visuelle proprement dite (blench dial. 'to glance'), soit l'émission de luminosité par un objet extérieur (blaze : « to burn with a bright fervent flame » $O E D$ ).

\subsubsection{Pour les adjectifs}

Ils désignent très souvent des couleurs (black, blue, et de très nombreuses formes dialectales, comme blae); ou qualifient les yeux (blind, blear). L'étude des 'mots en bl-' désignant des couleurs fait l'objet d'une étude séparée, voici un résumé succinct de nos conclusions : d'une part, toutes les couleurs, hormis le vert, sont représentées par au moins un 'mot en bl-'. Par ailleurs, à l'exception de black et blue, les teintes désignées sont souvent non uniformes, difficiles à cerner, composées de plusieurs couleurs entremêlées. De plus, non seulement règne la polychromie au niveau des teintes, mais la polysémie au niveau des termes (ex : bloom EDD). Cependant, en dépit de cette apparente complexité, nous notons deux regroupements, l'un autour de «blanc/pâle/clair », et l'autre autour de "sombre ». Enfin, afin de rendre compte de ces deux pôles opposés, ainsi que de black et de blue, nous avons conclu que ces mots proviennent vraisemblablement de formes dont le sens signalait plus le caractère lumineux et brillant d'un référent, qu'une couleur particulière.

\subsection{Délimitation de deux « Domaines Notionnels »}

Nous nous inspirons de la Théorie des Opérations Enonciatives proposée par Culioli pour ce qui est du concept de 'notion'. Dans l'ensemble du 'foisonnement' dont fait mention Culioli, il nous paraît possible de préciser quelques propriétés constitutives des deux domaines notionnels que nous examinons ici: FLOT / COURANT et VISION / LUMINOSITE. 


\subsubsection{Notion de FLOT / COURANT}

Deux caractéristiques nous semblent dignes d'intérêt: dans la production du courant, l'action de la bouche et des lèvres est déterminante ; cependant, il peut aussi souvent s'agir de phénomènes météorologiques (blast). En outre, les substances transportées ou projetées sont aussi bien de l'air que du liquide, voire un mélange d'air et d'eau : en particulier pour les verbes désignant des manières de pleurer (blirt «to cry, weep, shed tears »; ou le participe passé blubbered " tear-stained, disfigured by weeping »), il est difficile de distinguer ce qui l'emporte dans l'appréhension du procès, des flots de salive, de larmes, de sécrétions nasales, ou des hurlements. Avec le souffle qui s'échappe de la bouche, sont également émis des paroles ou d'autres sons, plus caractéristiques de l'animal que de l'homme la plupart du temps (blab : « to make a gurgling sound with the lips » $(E D D)$ ).

Lorsque cette notion est actualisée sous la forme d'un verbe, le caractère relationnel propre au verbe ainsi que la prise en compte de la dimension temporelle permettent une appréhension de l'agent et surtout du résultat dans la construction du sens. Par exemple, le référent est envisagé dans sa globalité pour bladder n., et le verbe correspondant bladder « to swell out like, or into, a bladder ; to inflate » $(O E D)$ profile en filigrane le rôle de la bouche comme agent, ainsi que son résultat, la formation d'un réceptacle susceptible de se remplir.

\subsubsection{Notion de VISION / LUMINOSITE}

Elle renvoie à l'idée générale de lumière : l'émission de rayons lumineux tout autant que sa perception par l'organe de la vue, l'œil. Si l'on considère le procès dans son ensemble, on va toucher à certaines caractéristiques de l'agent, c'est-à-dire la source lumineuse (qui peut fréquemment s'apparenter au feu), puis au déroulement du procès, et enfin à ses conséquences ou son résultat, c'est-à-dire la sensation visuelle proprement dite. Le même procès pouvant être envisagé à différents moments, il n'est donc pas étonnant que, si on tient compte à la fois du regard aspectuel et du cadre temporel, les manifestations sémantiques de cette notion pour une même forme, blaze par exemple, soient des sens aussi différents que « to burn with a bright fervent flame » (source et déroulement), " to shine brightly, to glitter » (déroulement), et « $(O b s$.$) to dazzle or daze with light; fig. to blind» (O E D)$, qui profile le résultat de l'action, et même le résultat indirect, à long terme.

\subsection{Les deux sous-classes de 'mots en $b l$-'}

Nous avons observé que $45 \%$ des 'mots en $b l$-' ont au moins un sens renvoyant à la notion de FLOT / COURANT, et 32,5\% à celle de VISION / LUMINOSITE, c'est-à-dire que plus des trois quarts de l'ensemble des 'mots en $b l$-' sont concernés. Nous identifions au sein de la classe constituée par les 'mots en $b l$-' deux sous-classes dont chacune est composée de lexèmes possédant au moins un sens qui renvoie à l'une de ces notions. 


\subsection{Cas édifiants de polysémie}

\subsubsection{Cas renvoyant à des correspondances macrocosme / microcosme}

Un même 'mot en bl-' peut désigner soit une rafale de vent, soit la production d'un souffle d'air par la bouche, et parfois le nez (blast n., blirt dial). Le souffle d'air ou le jaillissement de liquide proviennent donc soit des éléments (souvent déchaînés) de l'univers, soit du corps humain (bouche, nez, yeux). Ainsi, la citation fournie par l'OED «broken by the blast of a hurricane » a-t-elle trait au macrocosme (sens $n^{\circ} 1$ : «a blowing or strong gust of wind »), alors que l'extrait biblique "With the blast of thy nostrils the waters were gathered together " (Ex. xv. 8) correspond au sens obsolète faisant intervenir le microcosme : " A blowing of air through the mouth or nostrils; a breath ».

\subsubsection{Cas témoignant de l'exercice de plusieurs modalités perceptuelles}

Le même terme peut désigner des référents différents, correspondant à des perceptions relevant de modalités perceptuelles différentes, souvent l'ouïe et la vue (blare $\mathrm{n}$.). Le glissement de l'ouïe à la vue est indiqué explicitement dans les traits définitoires de blatant, (« in recent usage, obtrusive to the eye (rather than to the ear as in orig. senses)» OED). De même pour blind : l'OED indique non seulement l'évolution de "privé de la vue » à "privé de toute perception », mais indique pour le sens $n^{\circ} 14:$ : (Obs.) transferred from sight to sound ». Le lexicographe lui-même mentionne une certaine part de réalité extralinguistique comme étant à l'origine de ces évolutions sémantiques et de ces cas de polysémie. Nous pouvons citer en contexte "With a great thump of timpani and a blare of brass, the whole band come sweeping in » (ouie), et " The womenkind did not put on much 'blare' of color » (vue) (citations fournies par l'OED).

\subsubsection{Cas soulignant les relations qu'entretiennent vision et luminosité}

Plusieurs termes obsolètes dénotent à la fois le rayonnement provenant de l'œil ('glance', 'look') et celui émis par l'objet considéré ('shine', 'gleam') : il s'agit de blush (n., v.), blenk, blink $(O E D)$, et blinter $(E D D)$. Le rayon lumineux semble donc envisagé dans les deux sens, de l'œil à l'objet et/ou de l'objet à l'œil ; et nous comprendrons mieux l'importance de ce cas de polysémie lorsque nous évoquerons la théorie platonicienne de la vision. La définition de blind n. est d'ailleurs particulièrement explicite : "anything which obstructs the light or sight »: on envisage ici à la fois un rayon 'entrant' et un rayon 'sortant'. Nous pouvons opposer le sens de blink dans " A brown setter lay upon the hearth rug, blinking at the blaze ", où s'exercent à la fois la perception visuelle et l'activité des yeux et des paupières (sens $n^{\circ} 4 . b$ " To look with twinkling eye-lids, as one half-awake or dazzled with light »), à celui qui se manifeste dans «The sun was blinking on the windows », où l'agent n'est plus l'œil, mais une source lumineuse extracorporelle (soleil, étoiles, feu, bougie). 


\subsubsection{Cas préfigurant les relations qu'entretiennent les deux notions}

Certains lexèmes ont la particularité d'être polysémiques selon le schéma suivant: l'un des sens renvoie à la notion $<$ Flot/Courant $>$, alors que l'autre (ou d'autres) renvoie(nt) à $<$ Vision/Luminosité $>$. Par exemple, plusieurs formes, en général obsolètes, désignent à la fois une rafale de vent, un souffle, ou le jaillissement d'un liquide ; et, d'autre part, un éclair, une lueur, l'éclat du feu etc. Citons par exemple blast, blash, blare, blirt, et blizzener (dial.). De plus, à ces cas de polysémie s'ajoutent plusieurs mots traités comme des homonymes par le lexicographe (blaze $\mathrm{v}^{1}$ et blaze $\left.\mathrm{v}^{2} O E D\right)^{12}$. Enfin, des sens renvoyant à l'une des deux notions dans le lexique actuel renvoient à l'autre en dialecte (bleach, blear, blaze, blush, bleak). Par exemple, le sens actuel de bleach met en jeu la vision («To whiten by washing and exposure to sunlight or by chemical processes », OED), alors que l'EDD atteste des sens ayant trait à la force du vent et/ou de la pluie, comme dans l'énoncé : Nhb. «[My roof] is aal tight, except when the rain bleaches ». De même, bleak peut désigner une couleur en dialecte («pale, wan, sickly-looking » $E D D$ ), comme dans la phrase : «I can't justly say as she were well. She were looking so wankley and bleak » $(E D D)$; au contraire les sens suivants renvoient à la notion de Flot/Courant: «The cold wintry wind blew bleak »; "Scarce a shed to shelter me from the bleak air »; « Bleak hills and leafless woods » $(O E D)$.

\section{5. 'Mots en bl-' et parties du corps}

\subsubsection{Quelles parties du corps?}

\section{- Le sang - blood}

- La vessie - bladder

- L'œil - une forme dialectale: blinker, attestée par l'EDD et l'OED, avec la mention « argotique »; blinkers, ainsi que bliers, désignent aussi les cils (EDD)

- La lèvre inférieure - blob: attesté dans l'OED avec le qualificatif "pouting », et dans l'EDD : «cf. blubber-lipped : having thick-lips »

- Les os plats : blade : "A broad flattened bone or part of bone », en particulier le nom composé shoulder-blade 'omoplate'

- La graisse : blubber: L'OED n'atteste pour le nom ${ }^{13}$ que le sens actuel de «the fat of whales or other cetaceans ». Webster' $s^{14}$, et plusieurs dictionnaires bilingues, donnent un sens dérivé à nuance familière : « superfluous fat on a person or animal »

- Vésicules, cloques et autres pustules - l'un des noms usuels correspondant à ce sens est blister : "A thin vesicle on the skin, containing serum, caused by friction, a burn, or injury » $(O E D)$. Dix-sept noms au total désignent des lésions cutanées souvent renflées, contenant un liquide susceptible de s'écouler. Citons blain: "a sore, an ulcer or gathering of any kind; a swelling » $(E D D)$.

En outre, bien que non désignées par un 'mot en $b l$-', cinq parties du corps interviennent presque systématiquement dans les procès désignés par les verbes : la bouche, les yeux, et

\footnotetext{
${ }^{12}$ Blaze : la locution verbale blaze abroad est toujours attestée dans le dictionnaire bilingue Robert \& Collins (1998, $5^{\text {ème }}$ éd.), avec le sens de 'crier sur tous les toits'.

${ }^{13}$ Blubber (n.) : Sous la même entrée, l'OED atteste aussi les sens obsolètes de «The foaming and boiling of the sea », «A bubble of foam or air upon water », ainsi que «A jelly-fish or Medusa, also called sea-nettle », et "The action of blubbering or weeping ».

${ }^{14}$ Webster's Third New International Dictionary, 1993, Springfield (Mass.) : Merriam Webster Inc.
} 
moins fréquemment, les oreilles, le nez, et la peau. Notons que ce sont des lieux où s'exercent les sens, mais ces parties du corps sont ici souvent envisagées en tant qu'orifices, situés sur le visage, par lesquels s'écoulent divers flots de larmes, de souffle, de salive, ou de paroles. La partie du corps concernée englobe parfois la partie contiguë, par exemple les paupières. Le lexicographe ne s'embarrasse pas de précisions superflues et indique pour blink (sens 3 à 6) : "In various shades of meaning which run into one another: in the earlier, the notion of 'glancing' predominates, in the later, that of 'winking' » $(O E D)$.

Ces références peuvent être très explicites, voire insister sur le rôle précis joué par ces parties du corps (blabber: " to make sounds with the lips and tongue as an infant » $O E D$ ), mais celles-ci interviennent aussi de façon implicite dans les procès désignés. En ce qui concerne les verbes renvoyant à la notion $<$ Courant/Flot $>$, l'agent sera implicitement la bouche, et parfois le nez. Pour $<$ Vision/Luminosité $>$, l'agent, même implicitement mentionné, sera l'oeil. Enfin, pour des sens tels que « to burst out into weeping » (blurt, OED), les yeux et la bouche sont agents du procès, et les oreilles, ainsi que les yeux, organes de la perception.

\subsubsection{Caractéristiques}

\section{- Eruptions cutanées}

Les parties du corps désignées par des 'mots en bl-', sauf blood, bladder, blinker et blob dans le sens de 'lèvre inférieure protubérante' sont des lésions cutanées : dans la plupart des définitions, la peau est implicitement ou explicitement mentionnée. Elles sont perceptibles soit visuellement, soit par la sensibilité haptique, ou plus exactement par la douleur qu'elles provoquent. Elles attirent principalement l'attention visuelle soit par leur couleur (rouge ou blanc), soit par la forme saillante qu'elles dessinent à la surface de la peau.

- Air et eau

Ces cloques et boutons contiennent souvent une sérosité, susceptible de s'épancher hors du corps, et mentionnée dans les définitions, comme par exemple, blister : «A thin vesicle on the skin, containing serum » $(O E D)$. La présence de liquide dans ces vésicules semble une constante, indiquée de manière explicite ou implicite. Le sens usuel de bladder est éloquent à cet égard : "The musculo-membranous bag which serves as the receptacle of the urinary fluid secreted by the kidneys » $(O E D)$. Si ces cloques ne contiennent pas de liquide, ce sont des vésicules ayant la forme de petits sacs gonflés d'air. Parfois même, un processus de fermentation est à l'œuvre, susceptible de résulter en l'émission de produits gazeux.

En outre, à l'exception de la salive, tous les fluides corporels sont soit désignés par un 'mot en bl-' (blood; blee : «a tear»EDD), soit mentionnés dans certaines définitions. Nous venons de citer celle de bladder, pour l'urine ; ajoutons les larmes (blather : « to besmear with mud, blood, or tears »; bleary EDD), la sueur (blowze EDD), les mucosités bronchiques (blathery $E D D)$, et les sérosités sous-cutanées (blawp EDD).

\subsubsection{Premières conclusions : vers une approche cognitive}

\section{- Liens avec le sang}

De prime abord, les caractéristiques que nous avons dégagées pour la grande majorité de la sous-classe des 'mots en bl-' désignant des parties du corps (19 mots sur 23), semblent difficilement pouvoir s'appliquer à l'un de ses membres les plus courants en anglais moderne, à savoir blood. Nous atteignons là les limites de l'analyse sémantique traditionnelle; et une 
approche prenant en compte les fondements conceptuels à l'origine de la structure linguistique parait justifiée: "The main reason for adopting this procedure is that some linguistic forms are meaningless if taken literally, but they can be accounted for if reference is made to the cognitive factors that can be held responsible for their growth » (Heine 1997: 16).

- Sens de blype

Le sens d'un 'mot en $b l$-' a attiré notre attention eu égard à l'éventualité d'une approche cognitive. Il s'agit de blype : " a pellicule, a shred » $(E D D)$; « a shred of skin » $(O E D)$. Il semble difficile d'en rendre compte hors du cadre proposé par une analyse prenant en considération les représentations mentales basées sur les propriétés extralinguistiques du référent : nous pourrions alors rapprocher les conceptualisations des sécrétions s'écoulant du corps, et ces petites squames qui, également, se détachent des téguments humains.

\subsection{Aspectualité}

\subsubsection{Paroxysme et excès}

Dans les traits définitoires, les adjectifs et adverbes employés soulignent la plupart du temps la force, l'intensité, la violence des phénomènes désignés par les 'mots en $b l$-'. Par exemple, Blast : «a strong gust of wind »; sens $n^{\circ} 10$ de blast v. : « to strike with the wrath and curse of heaven » $(O E D)$. S'il s'agit de langage ou de cris, ceux-ci sont stridents : Blatant : " offensively or vulgarly clamorous » $(O E D)$; s'il s'agit de luminosité, celle-ci est si intense qu'elle peut en devenir aveuglante (blaze v1 : Obs. « to dazzle or daze with light; to blind » $O E D$ ). Les qualificatifs signalent atteinte et agression; et le résultat en est le mécontentement, la désapprobation, ou la douleur.

\subsubsection{Mélange et désordre}

Les couleurs ${ }^{15}$ désignées par des 'mots en $b l$-' sont souvent entremêlées et diverses ; de même les substances sont composites, les phénomènes confus et désordonnés. Par exemple, les rafales sont souvent composées de vent et de pluie, parfois mêlées de neige ou de grêle. Nous connaissons le sens de blend « to mix, mingle »; en dialecte blanda désigne un mélange d'orge et d'avoine semées ensemble $(E D D)$, blanch le minerai de plomb mélangé à d'autres minéraux $(E D D)$, et blewitt un cataplasme composé de quatre plantes et de pain $(E D D)$.

Les verbes désignant des façons de parler signalent l'absence de maîtrise, le manque de cohérence et d'organisation : Blab : «one who has not sufficient control over his tongue; loose talk or chatter; babbling » $(O E D)$. Les expressions « fitfully », ou " by fits and starts » se rencontrent à plusieurs reprises dans les définitions, et indiquent le caractère saccadé, impulsif, et irrégulier des procès, y compris lorsque l'agent n'est pas humain (blink: « to shine fitfully, to flicker », EDD).

\footnotetext{
${ }^{15}$ Rappelons que les mots désignant des couleurs ont fait l'objet d'une autre étude de notre part (Communication Atelier des Doctorants - Congrès SAES Nantes, 12 mai 2006).
} 


\section{Analyse étymologique}

\subsection{Racines indo-européennes homonymiques : *bhel-}

Trois des quatre racines indo-européennes ayant la forme *bhel- identifiées par le dictionnaire étymologique de Watkins sont à l'origine de la plupart des 'mots en $b l$-' que nous examinons ici. Il s'agit de :

${ }^{*}$ bhel ${ }^{1}$ " To shine, flash, burn; shining white and various bright colors », dont divers élargissements germaniques ont donné par exemple bleach, bleak, blaze, blind, blank, et la plupart des mots désignant des couleurs (black, blue, blond, blush)

${ }^{*} \boldsymbol{b h e l}{ }^{2}$ « To blow, swell ; with derivatives referring to various round objects and to the notion of tumescent masculinity »; dont les élargissements, selon différents itinéraires (extensions en *bhle- ${ }^{2}$, *bhlei-, ou *bhleu-) ont donné blow, bladder, blast, blain, blister, bloat

* bhel- ${ }^{4}$ « To cry, yell » dont l'extension ${ }^{*} b h \bar{e}^{-}{ }^{1}{ }^{1}$ « To howl » est à l'origine de bleat et blare

\subsection{Cas de blood}

Mallory \& Adams identifient deux racines que continuent la plupart des mots désignant le sang dans les principales langues issues de l'indo-européen : l'une, ${ }^{*} h_{1} e ́ s h_{2} \bar{o} r$ aurait désigné le sang coulant dans les veines, l'autre *kréuh $h_{a}$, le sang à l'extérieur du corps, le sang s'écoulant d'une blessure. " It has been argued that the lexical distinction between '(inside) blood' and '(outside) blood' in PIE is emphasized by derivatives and extensions of meaning that indicate two opposed metaphorical sets. [...] the root for '(outside) blood' yields words signifying aggression (e.g. Lat. crüdèlis 'cruel') [...], and dying, seen metaphorically in terms of the hardening (or freezing) of 'outside blood' [...]» (Mallory \& Adams 1997 : 71).

A cette conception 'négative' du sang 'à l'extérieur du corps' s'oppose la vision 'positive' du sang coulant dans les veines, symbole de vie et de fertilité masculine : "*ēs-r- 'inside blood' alludes to the growth of life and the warmth of spring » (Linke $1985: 341$ ). "There is some evidence that Indo-Europeans conceived of ${ }^{*} \bar{e} s-r$ - 'inside blood' as 'good'. When this blood penetrated the confines of the body, flowing toward the outside, it became *kreu-, a 'maleficient, evil' fluid » (ibid. : 343).

Cependant, blood et sa lignée germanique ne dérivent ni de l'une, ni de l'autre de ces racines ; et les dictionnaires étymologiques ne proposent en général qu'une racine germanique (*blöðam), et non indo-européenne, de blood. C'est le cas d'Onions (Onions 1966 : 101), de Mallory et Adams (M \& A 1997 : 71), ou de Chantraine : «Il n'y a pas de nom du sang commun à tout l'indo-européen" (Chantraine 1999: 34). Seuls certains "linguistes intrépides » ont l'audace de proposer une racine indo-européenne ${ }^{16}$ : ainsi, selon Watkins, le mot blood est issu de la racine indo-européenne * hel- $^{3}$ 'to thrive, bloom' (dont sont issus en anglais bloom, blossom, et blade), et non de la racine homonymique *bhel- ${ }^{1}$ 'to shine, flash, burn; shining white and various bright colours' (Watkins 2000: 9). Buck partage partiellement cet avis, et ajoute l'idée du jaillissement hors du corps : «OE blōd, etc., general Germanic, probably as 'that which bursts out' » (Buck $1949: 206$ ).

\footnotetext{
16 «Des linguistes intrépides le relient par une suite de chaînons sémantiques au nom de la fleur, allemand Blume, anglais bloom, etc., qui lui, est sûrement un cousin du latin flôs. Mais les chaînons sont subtils. En tout cas, le mot est isolé ; le groupe germanique, comme il est fréquent, fait bande à part » (Georges Dumézil, 1983 : «Le sang dans les langues classiques », Nouvelle revue française d'hématologie vol. 25, pp. 401-404).
} 


\subsection{Cas de spécialisation ou de généralisation sémantiques}

A travers les lexiques de diverses langues issues de l'indo-européen, les dictionnaires étymologiques consultés font état de traces qui, selon eux, témoignent de correspondances entre Vision et Lumière d'une part, Aspect et Vision d'autre part, et esquissent certaines manières d'envisager les fluides corporels comme 'mouvements de sortie' du corps humain.

\subsubsection{Vision et Luminosité : de 'soleil/œil' à 'briller/voir'}

Sous l'entrée 'eye', Mallory et Adams font état du reflet que renvoie le lexique de certaines langues issues de l'indo-européen des croyances ${ }^{17}$ associant un élément de l'univers à une partie du corps humain: "In IE cosmology, the concepts of 'eye' and 'sun' are constantly linked to one another, and in at least one case, O.Ir. süil 'eye', the word for 'eye' derives from the word for 'sun' » (M \& A 1997 : 188). De même, l'émission de lumière est l'une des sources principales que recense Buck à l'origine des mots désignant l'œil : «Most of the words for 'eye' belong to an inherited group [< IE * ok $\left.k^{w}-\right]$. The others are from such notions as 'see, look, bright, sun (?), shining ball' » (Buck 1949 : 225).

Afin de rendre compte de l'évolution sémantique des verbes concernés ('briller' > 'voir'), Buck conjecture un cheminement passant par le relais des noms signifiant 'lueur, éclair', qui, lorsqu'ils s'appliquent au regard, permettraient l'émergence des sens 'voir, regarder' : "The dependence of sight upon light is reflected in several words for 'see' and 'look' that are cognate with others for 'light, shine' etc. » (Buck 1949 : 1041). C'est le cas, entre autres, du rameau germanique, et plus précisément, de certaines formes en bl-: «NHG blicken [...] 'look' (also 'gleam, shine'), from [...] OHG blicchen 'gleam, shine', [...] whence NHG blick n. 'glance, look', and through this the use of the verb in sense of 'look' » (Buck 1949 : 1044).

Ces cas de spécialisation sémantique, constatés dans les évolutions des sens de mots appartenant à diverses langues issues de l'indo-européen, viennent étayer les observations notées à ce propos dans notre analyse sémantique des 'mots en $b l$-' du lexique anglais, à savoir la polysémie briller/voir des cinq formes blenk, blink, blinter, et blush $\mathrm{n}+\mathrm{v}$. , la polysémie de blind adj. " destitute of the sense of sight / enveloped in darkness ; obscure ; Obs. not lighted », et la définition de blind $\mathrm{n}$. : « anything wh. obstructs the light or sight ».

\subsubsection{Vision, Aspect général, et Couleur}

Une seconde extension sémantique, dont témoignent plusieurs 'mots en $b l$-', en général dialectaux (bloom, blush), est attestée par Buck dans le même paragraphe : l'émergence pour les noms du sens "apparence extérieure, aspect», et, pour les verbes, de "paraître, avoir l'air », processus affectant des formes dont le sens originel renvoie à la notion de Vision. Buck qualifie ce changement sémantique de « relation logique »; il indique même l'origine probable du lien en employant "subjective » pour le sens attaché à 'sight, vision', et « objective » pour celui de 'look, appearance'. Par l'emploi de ces deux adjectifs, Buck évoque le rayon imaginaire associant le sujet voyant à l'objet vu, rayon qui peut être qualifié de « visuel » dans un sens, et de « lumineux » dans l'autre : "Words for the subjective 'sight' are nearly all derivatives of the verb for 'see', or in some cases 'look'. Many of these are also

\footnotetext{
${ }^{17}$ Nous présenterons brièvement le système de croyances des Indo-Européens dans ce domaine en section 4.
} 
used for the objective 'look, appearance' of something [...]. This notion is logically related to the subjective 'see' or 'look' in the same way as the obj. to the subj. 'smell' or 'taste', and in part is expressed by forms of, or related to, the vbs. for 'see' or 'look' » (Buck 1949 : 1041).

Enfin, l'aspect général est parfois lié à la couleur (blee : "colour, complexion »; bly: «a faint likeness or resemblance, a 'look of' »EDD). L'OED, pour sa part, atteste trois sens pour blee : «1. Colour, hue ; 2 . Colour of the face, complexion ; visage ; 3 . Appearance, form », et conjecture une provenance du germanique. Les sens donnés dans l'OED sous bly témoignent de degrés plus avancés de généralisation : "Likeness, resemblance, look, aspect, species, character » $(O E D)$. Les liens qu'entretiennent la couleur et l'aspect extérieur d'un objet, en tant qu'entités les plus immédiatement perceptibles par la vue, sont reflétés, une fois de plus, par le lexique : "Within individual languages, terms for color often derive from terms for the 'surface', e.g., Lat. color (akin to cèlāre 'cover, conceal') [...] or are derived from a specialization of individual color terms. [...] The most interesting of these terms appear to relate to markings rather than color per se. A root*perk is applied to a variety of variegated beings, e.g., Lat. porcus 'perch or piglet' » (M \& A 1997 : 113). Un 'mot en bl-', bloached, a précisément ce sens : " of a variegated appearance, spotted » $(E D D)$. Buck est très explicite quant aux relations qui lient vue, couleur et apparence, et cite Aristote : "'Color' is treated by Aristotle as the objective parallel to the subjective 'sight', like 'sound' to 'hearing', etc. ; and while 'color' is only one aspect (beside 'form, shape') of an object's 'look, appearance' (the true obj. notion parallel to 'sight'), it is the most conspicuous and one depending wholly on sight. [...] Most of the words for 'color' reflect such notions as 'covering', 'surface, skin', 'countenance, look', or the 'hair' of animals » (Buck 1949 : 1050).

\subsubsection{Fluides corporels et mouvements de sortie du corps}

Dans les sections qu'il consacre aux mots désignant divers produits que le corps évacue, tels que l'urine, les excréments, les vomissements, ou la sueur, Buck observe des sens premiers qui renvoient à des représentations désignant ceux-ci comme étant des 'mouvements de sortie' du corps. Citons par exemple les remarques de cet auteur sous l'entrée " excrements »: " In the Germanic group and several of the other words the development is from 'separate', whence 'discharge from the body'. The countless euphemistic expressions are mostly ignored, as for example those meaning literally 'discharge, empty one's self, ease one's self [...]' » (Buck 1949 : 275).

Les produits corporels examinés ci-dessus ne sont pas directement désignés par des 'mots en $b l$-'. Mais les observations des étymologistes sont extrêmement éclairantes quant aux possibles conceptualisations que les mots les désignant recouvrent. Rappelons enfin que blood et les formes apparentées du rameau germanique, qui se distinguent comme ne provenant pas des deux racines indo-européennes de loin les plus répandues ( ${ }^{*} \bar{e} s e n-$ et $\left.* k r e w-\right)$, sont attestés par Buck comme issus d'une forme germanique à l'origine également de blade, bloom et blossom en anglais moderne, et dont le sens paraît être : « ce qui jaillit » (Buck 1949 : 206). 


\section{Dimension mythique : réprésentations du corps chez les peuples anciens}

\subsection{Corps et cosmos}

Dans la tradition indo-européenne, le premier homme provient de la mise à mort, suivie du démembrement, d'un être ou géant originel, dont les différentes parties du corps sont utilisées pour créer les éléments constitutifs du cosmos : "The general narrative is that a primordial being is killed and dismembered, and that from that being's body the cosmos or some important aspects of it are created (from the pieces of the victim's body) » (Lincoln 1986 : 2-3). A leur tour, ces parties de l'univers, une fois assemblées, permettent la création de l'homme, dont chacune des parties du corps est liée à un élément du cosmos. Des neuf homologies qui président aux correspondances reliant les parties du corps aux éléments de l'univers, nous retiendrons les trois suivantes pour notre propos: Souffle / Vent; Yeux / Soleil ; et Sang (ou autres fluides corporels) / Eau. Ces correspondances ne reposent pas sur de simples similitudes apparentes: parties du corps et parties de l'univers correspondantes sont des avatars d'une seule et même substance, et apparaissent en alternance dans le cycle de la vie et de la mort, dans une transmutation perpétuelle.

\subsection{Le souffle et le vent}

L'homologie qui fait correspondre le souffle humain au vent de l'univers est l'une des plus récurrentes du système ; le cycle respiratoire de l'homme étant identifié au processus respiratoire du cosmos : au cours de l'expiration, le souffle quitte le corps et se métamorphose en vent, et l'inverse se produit lors de l'inspiration. "The simple act of breathing thus alternately constructs the macrocosm (while deconstructing the microcosm), then constructs the microcosm (while deconstructing the macrocosm) » (ibid. : 33-34).

\subsection{Eil, soleil, lumière et paroles}

\subsubsection{Croyances indo-européennes : homologie Yeux / Soleil}

De même, parmi les neuf homologies qui structurent le système de pensée indo-européen, celle faisant correspondre les yeux au soleil est l'une des plus solidement établies. Lincoln signale une particularité du système de pensée indo-européen : l'œil et le soleil sont tous deux des sources de lumière qui, conjointement, entrent en jeu dans le mécanisme de la vision (Lincoln $1986: 17-18$ ).

\subsubsection{Grecs de l'époque homérique : les « souffles »}

Pour les 'Premiers Grecs', si les oreilles, les yeux, et le nez sont bel et bien reconnus comme les organes des sens, la perception dans son ensemble est saisie en fin de compte dans le thumos $^{18}$, et les phrenes $^{19}$. Le cheminement des sensations, tel que les Grecs l'imaginaient,

\footnotetext{
${ }^{18}$ Thumos $(\theta v \mu o ́ \varsigma)$ : souvent considéré comme la substance de la pensée et de la conscience; identifié au 'souffle', qui représentait la conscience dans la poitrine. Souvent rendu par 'âme-souffle', anéantie par la mort, par opposition à psukhe, immortelle, et extérieure au moi conscient.
} 
aboutit aux poumons, lieu ultime de destination des sons, des images visuelles et des odeurs (Onians 1999 : 93-96). Les trois fonctions que sont la vision, l'audition, et l'olfaction sont donc envisagées comme des souffles émis par les objets, et pénétrant dans le corps par l'intermédiaire de ce que est identifié de nos jours comme 'les organes des sens'.

\subsubsection{Vision, lumière, et langage : entrées et sorties du corps humain}

Il nous reste à examiner une dernière particularité propre aux 'souffles' : leur direction. Selon d'autres théories plus tardives de la vision chez les Grecs, un double mouvement semble se produire entre l'extérieur et l'intérieur du corps : «[...] les yeux ne reçoivent pas seulement la lumière ou ce qui est 'soufflé' par les objets, ils sont eux-mêmes désignés comme des sources de même type [...]. Chez Homère, survit la croyance que le feu flambe dans les yeux, surtout lorsqu'on est en colère » (Onians 1999: 101). Si nous nous tournons vers Hésiode, nous retrouvons une conception similaire du 'feu du regard', envisagé comme substance sécrétée par le corps et excrétée par l'œil.

En outre, les paroles, chez Homère, sont souvent qualifiées 'd'ailées', car elles sont véhiculées entre le locuteur et l'auditeur par le souffle qui traverse l'air ambiant: «Elles sortent avec le souffle qui est l'intelligence en elles, elles sont des parties du souffle, et l'auditeur les prend et les met dans son thumos » (Onians 1999: 90-91). En ce qui concerne ces conceptualisations qui envisagent globalement les mouvements d'entrées et de sorties du corps, et associent à ce titre le langage, en tant que flot de paroles, aux sens, nous noterons que ces mouvements de va-et-vient s'effectuent grâce à des passages qui ne sont autres que la bouche, le nez, les yeux et les oreilles.

\subsubsection{Théorie platonicienne : transmission de «mouvements »}

Dans l'Antiquité, deux grandes théories de la vision s'affrontent : pour les théories dites 'de l'émission', l'œil émet des rayons visuels ; c'est le jaillissement du feu contenu dans l'œil qui permet de voir. Au contraire, pour les théories 'de l'émanation', « il y aurait un flux corpusculaire ténu qui va de l'objet à l'œil de l'observateur » (Imbert 2005 : 91-93).

Platon fait une synthèse des théories de l'émission et de celles de l'émanation pour élaborer ses propres conceptions de la vision. L'œil devient le lieu de passage du «feu intérieur » qui va à la rencontre des effluves émises par l'objet : de la chose à percevoir part un rayon, ou plus précisément 'une effluve qualitative' qui, pour qu'il y ait vision, doit s'unir au rayon lumineux émanant de l'œil. "Selon Platon, affections, qualités et sensations sont des mouvements » (Timée : 242, note 267), que les organes des sens acheminent jusqu'à l'âme afin qu'elle acquière la connaissance du monde sensible. Le fondement du mécanisme régissant la sensibilité dans son ensemble, et la perception par les organes des sens, réside selon Platon en la transmission de mouvements qui s'effectuent entre le milieu et l'organisme, puis à l'intérieur de celui-ci.

\footnotetext{
${ }^{19}$ Phrenes ( $\left.\varphi \rho \varepsilon ́ v \varepsilon \varsigma\right):$ rendu traditionnellement par 'diaphragme', à tort selon Onians (1999), souvent considéré comme le centre des émotions et de la pensée. L’homme pense ses pensées et sent ses émotions en trois lieux : le cœur, les phrenes, et le thumos, tous situés dans sa poitrine.
} 


\subsection{Sang et fluides corporels}

Pour les Indo-Européens, l'homologie associant macrocosme et microcosme fait en général correspondre au sang les éléments liquides de la nature, le plus souvent le système fluvial, mais aussi la mer, la rosée, ou encore la pluie. Cependant, l'association avec l'eau de la nature n'est pas l'apanage du seul sang de l'organisme : l'ensemble des fluides corporels peuvent correspondre à cet élément, comme autant de flux susceptibles de s'écouler du corps. D'ailleurs, ces conceptualisations qui englobent les liquides de l'organisme évolueront pour constituer ultérieurement le système des quatre humeurs.

Nous retiendrons que l'idée maîtresse sous-tendant la représentation du sang chez Platon est celle d'un fluide aux multiples fonctions de transmission et de déplacement, au sein d'un réseau complexe de circulations internes au corps. En effet, à aucun moment le philosophe n'envisage le sang comme fluide susceptible de s'écouler hors de l'organisme; le sang est conçu dans le cadre des grands systèmes physiologiques. Cependant, en fin de compte, ses rôles s'inscrivent bien dans celui du mouvement général animant le cosmos : le sang est le fluide vital dont le mouvement assure la survie de l'organisme, face aux mouvements de déplétion qui l'assaillent, venus de l'univers: "Ces mouvements internes véritables, appartenant à l'organisme humain animé, trouvant leur source dans l'âme et allant de l'âme au corps, anéantiront les mouvements externes » (Joubaud 1991 : 228).

\subsection{Conclusions : mouvements entre corps et cosmos}

La première idée maîtresse qui sous-tend ces représentations paraît être le fait que l'homme n'est pas envisagé seulement en tant qu'organisme indépendant, mais en tant qu'occupant une place au sein de l'univers. Les rapports qu'il entretient avec le milieu, et plus globalement le cosmos, sont, de façon récurrente, déterminants dans la façon dont le corps est conçu. Le corps humain, et les parties du corps, sont donc conceptualisés en premier lieu à partir des relations et des correspondances que l'esprit, à tort ou à raison, établit entre ceux-ci et les éléments de l'univers.

La seconde grande composante de ces représentations est l'une des conséquences de la première idée force : elle a trait à la notion de mouvement. Les rapports qu'entretiennent l'organisme et le milieu sont fréquemment décrits en termes de dynamique des forces. D'une part, le corps est conçu dans le cadre de son environnement, il évolue au sein de celui-ci, et le cinétisme est essentiel dans la façon dont les parties du corps émergent à la conscience. D'autre part, corps et milieu interagissent: l'organisme est pourvu d'orifices qui lui permettent de communiquer avec le monde qui l'entoure, ainsi que d'agir sur lui ; les fonctions corporelles et les facultés humaines sont conceptualisées en termes d'échanges, de déplacements, de flux qui mettent en relation les deux domaines. Tout semble se passer comme si le corps était globalement pensé comme un espace fini et discret, certes, mais dont la finalité ultime consisterait à tisser des liens afin que le monde entre en lui et que lui-même se mêle au monde. 


\section{Approche cognitive}

\subsection{De la projection conceptuelle aux réseaux métaphoriques}

\subsubsection{Projection conceptuelle métaphorique}

Afin de rendre compte de certains des cas de polysémie, nous nous proposons d'envisager les phénomènes à la lumière de l'approche de Lakoff et Johnson. Selon ces auteurs, notre système cognitif établit des liens entre un domaine source et un domaine cible : «The essence of metaphor is understanding and experiencing one kind of thing in terms of another $»(\mathrm{~L} \& \mathrm{~J}$ 2003 : 5). Ces liens ne reposent en aucune façon sur une relation 'objective' qui existerait entre le langage et le monde: «Metaphors allow people to understand one thing as another, without thinking the two things are objectively the same» (Sweetser 1990: 8-9). La projection métaphorique s'effectue en général d'un domaine concret vers un domaine abstrait, qui est appréhendé, structuré, et exprimé, dans les mêmes termes que le domaine source : par exemple, de nombreuses émotions sont conceptualisées en termes de feu ou de chaleur.

Nous avons noté qu'un même 'mot en $b l$-' pouvait désigner ou qualifier d'une part la production d'un souffle d'air ou de sons par la bouche (et parfois le nez), d'autre part une rafale de vent; ou bien encore l'émission de liquide par la bouche, et une averse de pluie (mêlée ou non de grêle ou de neige). Tels sont les cas de blast, blirt, blow, et de formes dialectales (blash, blather, blossom, blury, blusterly). Nous constatons le même type de polysémie pour bloom, blenk, blink, blinter, etc, cette fois-ci entre le regard d'une part, et la lumière émise par le soleil ou une flamme d'autre part. Nous pouvons envisager ces cas comme l'actualisation du processus de projection conceptuelle métaphorique d'un domaine source, le corps humain, vers un domaine cible, les phénomènes météorologiques, et plus généralement, les éléments du milieu naturel. Le mécanisme de métaphore conceptuelle permet à l'esprit d'utiliser les inférences faites ici dans le domaine du corps, concernant principalement les fonctions attachées à certaines parties du corps (bouche, yeux, nez) pour les réinvestir dans le domaine du temps (weather) : certains coups de vent ou éclairs seront ainsi conceptualisés et exprimés par les mêmes lexèmes que ceux désignant les émissions et sécrétions issues du corps.

A ce sujet, nous avons évoqué plus haut certaines croyances indo-européennes, associant de façon systématique une partie du corps à un élément de l'univers: aux yeux correspondaient le soleil, au souffle le vent, et aux fluides corporels l'élément liquide de la nature. La projection conceptuelle est l'un des 'modèles cognitifs' permettant à l'esprit de structurer la pensée et d'acquérir la connaissance à partir de l'expérience ; il semblerait qu'il existe des similitudes troublantes entre la stratégie opérée par l'esprit dont ces cas de polysémie pourraient être des traces au niveau linguistique, et celle à l'origine des conceptualisations des peuples anciens, associant corps et milieu.

\subsubsection{Réseau conceptuel métaphorique}

Dans leurs ouvrages, Lakoff et Johnson donnent de multiples listes d'expressions conventionnelles, qui révèlent l'existence de réseaux métaphoriques au travers desquels un domaine abstrait dans son ensemble est appréhendé et structuré en termes d'un domaine concret. Ainsi la métaphore conceptuelle $<$ Theories Are Buildings $>$ permet-elle de concevoir le domaine de l'argumentation en termes de construction d'un bâtiment, ce de façon 
systématique (Johnson 1987: 105). Dans Philosophy in the Flesh, les auteurs examinent comment sont appréhendées certaines "idées philosophiques fondamentales », telles que le temps, la causalité, l'esprit, le moi, et la moralité. Le temps, par exemple, est conceptualisé en termes d'espace, plus précisément en termes de déplacements et d'événements dans l'espace, directement perceptibles. La causalité est souvent conceptualisée en termes de forces et de dynamique des forces.

En ce qui concerne les deux sous-classes de 'mots en $b l$-' examinées ici, nous n'avons pas à proprement parler découvert de réseau métaphorique structurant un domaine abstrait. Cependant certaines définitions ont attiré notre attention : d'une part certains référents, de sens fort différents, ont la particularité commune d'avoir subi une transformation, ou de devoir leur existence à l'action du vent. Citons quelques formes dialectales de l'EDD, comme blakes (« droppings of cows" dung dried out for fuel »); bleach $\mathrm{n}$. (« a drying-ground used for 'bleaching' linen »); bleean v. (« to whiten linen by exposing it a little to the wind »); bloatherring ("a smoked, half-dried herring »); blittered («torn by the wind »); ou encore blossomed («said of cream becoming full of air whilst churning »). Et n'oublions pas que l'un des premiers sens de bleak est « exposed, wind-swept ».

D'autre part, des définitions de plusieurs 'mots en $b l$-' mentionnent l'action de puissances occultes et de formes de sorcellerie : Blast: «a tumour in some places attributed to witchcraft ; an infectious or malignant air » $(E D D)$; Blink: " to exercise an evil influence, bewitch; hence to turn anything sour » $(E D D) »$. La polysémie de blas («1. a blast, a breath ; 2. a supposed 'flatus' or influence of the stars, producing changes of weather » $O E D$ ), et celle de blight («1. any baleful influence of atmospheric or invisible origin [...] that suddenly blasts, nips, or destroys plants »; 4. any malignant influence of obscure or mysterious origin » $O E D)$, se laissent interpréter sur le plan cognitif en termes de projection métaphorique. Cette dernière permet l'émergence de sens renvoyant à l'action de puissances surnaturelles, identifiées à des souffles exhalés par des forces malignes.

Il semblerait que tout un réseau conceptuel métaphorique permette d'appréhender, de structurer, et d'exprimer, par l'intermédiaire d'une sous-classe de 'mots en $b l$-', un domaine que l'on pourrait nommer «Systèmes de Croyances », à partir de la représentation de l'action de l'air et du vent. Notons enfin que l'influence néfaste peut aussi être attribuée à un œil : le sens $\mathrm{n}^{\circ} 7$ de blink en témoigne : " to turn milk, beer, slightly sour [The origin of this use has been sought in the glance of an evil eye, the 'blinking' of milk being formerly ascribed to witchcraft ; also in the effect of lightning, since thunder generally 'blinks' milk] » $(O E D)$.

\subsection{Fondement métonymique de la métaphore : cas de synesthésie}

\subsubsection{Trait saillant de « Paroxysme / Impétuosité »}

Nous avons noté plus haut une caractéristique que présentent un grand nombre de traits définitoires : il est souvent fait mention du caractère violent ou excessif des phénomènes désignés. D'autre part, des cas fréquents de polysémie indiquent l'intervention de plusieurs modalités perceptuelles, parfois expressément soulignées par le lexicographe (blare). Par exemple, considérons le cas de blatant: la polysémie de ce lexème au niveau linguistique ( « noisy; offensively or vulgarly clamorous » > « glaringly conspicuous ; palpably prominent or obvious »), peut s'interpréter, sur le plan cognitif, comme la projection métaphorique établie par entre le domaine source de la perception auditive et celui de la perception visuelle, et plus généralement de la perception dans son ensemble. 
Rappelons que, pour certains courants ${ }^{20}$ de la linguistique dite 'cognitive', la projection métaphorique repose en général sur une motivation métonymique: l'esprit extrait ${ }^{21}$ une propriété saillante du domaine source, qui est ensuite réinvestie dans le domaine cible. En réponse aux objections de Taylor, pour qui la synesthésie est le seul cas où la métaphore n'aurait pas une motivation métonymique (Taylor 2003 : 138-139), Barcelona ${ }^{22}$ examine le cas des expressions 'loud colour' et 'sweet music'. Nous nous inspirons de sa démonstration pour blatant : le domaine cible (vision) est appréhendé en termes d'un de ses sous-domaines saillants, celui reposant sur la caractéristique du paroxysme et de l'excès, propre, au niveau de la perception, à attirer l'attention, même involontaire. Ce sous-domaine détermine le choix du domaine source (ouïe), appréhendé en termes du même sous-domaine du 'paroxysme'.

Cette projection métaphorique reposant sur une motivation métonymique, où le domaine source et le domaine cible sont tous deux appréhendés à travers l'un de leurs sous-domaines saillants, celui de 'Paroxysme/Violence', nous semble constituer sur le plan cognitif l'une des opérations permettant l'émergence des cas de polysémie correspondants au niveau sémantique, très nombreux pour les 'mots en $b l$-'.

\subsubsection{Mélange et intermittence, confusion et désordre}

Nous pourrions appliquer une démonstration similaire à propos d'une autre caractéristique très fréquemment indiquée dans les traits définitoires: celle de mélange et d'irrégularité. Nous en déduirions de même l'émergence de sens tels que " of a variegated appearance ; spotted » pour bloached (EDD). Mais nous touchons là à d'autres particularités de la perception visuelle, ayant déjà attiré notre attention lorsque nous avions noté la polysémie de lexèmes tels que bloom $(E D D)$ : « a rosy colour or flush of the face » / «plumage of a bird », ou celle de blush(ed) (EDD) : « stained » / « appearance, resemblance ».

\section{3. « Fictive Motion »}

\subsection{1. «Factive Stationariness » vs. « Fictive Motion »}

Il semble possible de rendre compte de nombreux sens pris par les 'mots en $b l$-' en invoquant un certain type de conceptualisation d'événements, que Talmy nomme 'Fictive Motion', conceptualisation selon laquelle l'appréhension d'une situation statique se fait à travers des formes et des constructions linguistiques exprimant le mouvement: "Most observers can agree that languages systematically and extensively refer to stationary circumstances with forms and constructions whose basic reference is to motion » (Talmy $2000: 104)$.

\footnotetext{
${ }^{20}$ Par ex. Taylor (2003 : 138-140); Heine (1997 : 140); Langacker (1987: 168-171) ; Kövecses (2000 : 159).

21 'Extraction' : nous proposons d'utiliser ce concept culiolien (Culioli 1990 : 182) pour décrire une opération comparable, mais située en amont des opérations énonciatives : opération cognitive qui consisterait, face à un référent donné dans un cadre spatio-temporel donné, à repérer un certain nombre de propriétés caractéristiques de ce référent, et à en extraire certaines propriétés saillantes pour les réinvestir soit dans le même domaine, soit dans d'autres domaines en les projetant sur d'autres référents notamment à des fins de dénomination. Nous reviendrons sur ce terme lors de l'évocation du 'modèle en réseau' de Langacker (section 5.5).

${ }^{22}$ «On the plausibility of claiming a metonymic motivation for conceptual metaphor», in Barcelona 2003, Metaphor and Metonymy at the Crossroads : 31-58.
} 
Talmy en distingue plusieurs catégories, dont l'une des plus récurrentes consiste à rendre par un verbe de mouvement (go par ex.) une scène où aucun déplacement n'a effectivement lieu, comme dans 'This fence goes from the plateau to the valley'. Ce type de manifestation du phénomène a été le plus étudié jusqu'ici ; il correspond, par exemple, à ce que Langacker désigne par 'Abstract Motion' (par ex., Langacker 2002 : 12). Selon Talmy, l'écart entre le sens littéral de l'expression linguistique et la réalité provient d'un schéma cognitif majeur, selon lequel, chez le même individu, deux représentations de la même entité coexisteraient, dont l'une, celle qu'il nomme «factive », serait jugée plus 'véridique' que l'autre : «Of the two discrepant representations of the same object, we will characterize the representation assessed $^{23}$ to be more veridical as factive and the representation assessed to be less veridical as fictive » (Talmy $2000: 100$ ).

\subsection{2. 'Radiation Paths'}

Parmi les divers cas de Fictive Motion que distingue Talmy, celui qu'il nomme «Emanation », et, au sein de cette catégorie, le phénomène décrit par "Radiation Paths », semblent correspondre à certaines expériences désignées par des 'mots en $b l$-': «The linguistic conceptualization of a radiation path is of radiation emanating continuously from an energy source and moving steadily away from it» (Talmy $2000: 111$ ). Trois processus distincts contribuent à constituer le procès dans son ensemble : la production et l'émission de la radiation par la source, le déplacement de celle-ci le long d'une trajectoire qui conduit à l'objet soumis au rayonnement, et la façon dont la propagation de cette énergie affecte l'objet. Cette conceptualisation du rayonnement correspond à une construction linguistique typique, dans laquelle le déplacement fictif est généralement rendu par un verbe du type shine suivi d'un groupe prépositionnel (prépositions de mouvement : into, onto, down onto etc.) (Talmy $2000: 112$ ).

Nous avançons l'hypothèse que 'les verbes en $b l$-' correspondants pourraient permettre, de façon immanente ${ }^{24}$, la conceptualisation des procès désignés comme autant de 'déplacements fictifs'. Nous pouvons ainsi citer dix verbes attestés dans l'OED : blare (Obs.), blash, blaze, blenk, blik(e), bliken, blink (sens 5 : « to shine with flickering light »), bloom (sens 4: «to glow with warm colour»), blush (Obs.), et blysne; auxquels s'ajoutent six formes dialectales de l'EDD: blent, blicant (adj.), blint, blinter, blirt, blunk. Les traits définitoires de ces verbes désignent la même réalité que celle qui répond aux 'feature values' mentionnées par Talmy ${ }^{25}$ (Talmy 2000: 106). Un second déplacement fictif associé est souvent l'émission de chaleur (bloom $(E D D)$ : « of the sun : to shine scorchingly, to throw out heat as a fire »). L'objet affecté par ce rayonnement est principalement l'œil, et le corps tout entier pour l'effet calorifique. La source prototypique du rayonnement est le soleil ou le feu : même bleach, ou bleak (Obs.), qui ont évolué vers d'autres sens en anglais moderne, ont dans leurs définitions une allusion à la lumière (aveuglante) du soleil : " to whiten by washing and exposure to sunlight » $(O E D)$. Les traits définitoires de certains lexèmes, dont le sens usuel pourrait paraître éloigné, permettent de les inclure dans cette sous-classe, car il y est fait

\footnotetext{
23 Talmy attribue à deux sous-systèmes cognitifs différents les deux représentations concurrentes, et à un troisième le jugement qui fait que l'une 'l'emporte' sur l'autre : «We presume that the two representations are the products of two different cognitive subsystems, and that the veradicality assessment itself is produced by a third cognitive subsystem whose general function is to generate such assessments » (Talmy $2000: 100$ ).

${ }^{24}$ La définition de blush, 'to shine forth', est assez révélatrice à ce sujet; de même que celles, obsolètes, de bliss 'blitheness of aspect toward others, «light» of one's countenance' et de blithe 'exhibiting kindly feelings to others', où les sentiments bienveillants semblent irradier de la personne.

${ }^{25}$ L'une de ces 'values' se définit ainsi : « The fictively moving entity is itself fictive » (Talmy 2000: 106).
} 
mention d'une source de lumière. Citons par exemple blend: " to make blind, permanently or temporarily e.g. with bright light » $(O E D)$, et blind v. : " to deprive of sight, by dazzling with bright light » $(O E D)$.

Dans un passage qui peut laisser sceptique, Talmy souligne que pour un énoncé du type The sun is shining into the cave, bien que des particules soient effectivement émises d'après les lois physiques, le mouvement du rayonnement n'est pas perceptible, et qu'il s'agit bien d'un déplacement fictif, pur fruit de la conceptualisation par l'esprit de l'expérience: " Although physicists may tell us that photons move from the sun to the irradiated object, we certainly cannot actually see any such occurrence [...] In other words, the so-conceived motion of radiation from the radiator to the irradiated must be fictive motion » (Talmy 2000 : 112). Enfin, bien que Talmy n'indique pas que la représentation de ce type de procès relève du phénomène de Fictive Motion, il semblerait que nous puissions nous situer dans ce cadre pour rendre compte des sens des multiples ${ }^{26}$ 'mots en $b l$-' désignant l'émission de cris ou de paroles, par l'homme ou l'animal, comme blare, blab, blabber, ou bleat. Par exemple, les traits définitoires de blart $(E D D)$, après « 1 . to bleat, bellow » et « 2 . to cry, roar », mentionnent explicitement un mouvement sortant (out), et implicitement un destinataire situé au terme de la trajectoire empruntée par les mots : «3. to scold»; «4. to let out a secret ».

En conclusion, le concept de Fictive Motion nous permet de jeter les bases d'une cohérence avec la sous-classe des 'verbes en bl-' renvoyant à la notion Vision/Luminosité : en effet, exception faite de blow, et de quelques autres termes désignant l'émission d'un courant ou d'un souffle (blast), procès pouvant se concevoir comme un mouvement factif, et non fictif, au cours duquel une quantité d'air est effectivement déplacée, les autres 'verbes en $b l$-' de cette sous-classe conceptualisent parole et cris comme des entités provenant d'une source (la bouche), et se déplaçant suivant une trajectoire qui va jusqu'à l'oreille de l'interlocuteur.

Une ultime remarque amusée concerne l'un des sens dialectaux de blare, attesté dans l'EDD : « to protrude the tongue (also the eyes)». La similitude des mouvements des deux parties du corps n'est qu'apparente, car s'il y a effectivement mouvement sortant dans le cas de la langue, celui-ci ne peut être que fictif pour les yeux. Nous rejoignons ici les remarques de Talmy concernant l'iconographie populaire, qui représente sous la forme d'un faisceau lumineux émis par les yeux du héros de bande dessinée certains pouvoirs surnaturels de celuici, ou rend l'expression métaphorique 'to look daggers at' ${ }^{27}$ par la représentation de poignards lancés de l'œil du personnage (Talmy $2000: 125$ ).

\subsection{3. 'Sensory Paths'}

Talmy envisage d'autres types «d'Emanation», dont l'un a trait à la perception sensorielle, et qu'il désigne par l'expression 'Sensory Paths' : "This type of fictive motion involves the conceptualization of two entities, the Experiencer and the Experienced, and of something intangible moving in a straight path between the two entities in one direction or the other » (Talmy 2000: 115). A l'origine de cette double direction du mouvement, deux conceptualisations sont possibles : soit le sujet de la perception (Experiencer) envoie une sonde (probe) qui se dirige vers l'objet (Experienced); soit celui-ci émet un stimulus qui affecte l'appareil sensoriel de l'observateur. Le lexique reflète ces deux éventualités : en anglais, see a pour sujet l'agent et pour complément d'objet l'objet repéré ; alors que show

\footnotetext{
${ }^{26}$ Nous comptons 21 entrées rien que dans l'OED : blare, blab, blabber, blarney, blat, blate, blaterate, blathe, blatter, blea, bleat, bleep, blether (blather, blither), blirt, blore, blub, blubber, blurt, bluster, bluther, et blutter.

${ }^{27}$ La traduction française, 'foudroyer quelqu'un du regard', participe de la même conceptualisation, et met en jeu le même phénomène de Fictive Motion.
} 
permet de conceptualiser l'objet perçu comme source de la sensation : «[...] show ${ }^{28}$ is lexicalized to take the Experienced as subject and can take the Experiencer as the object of the preposition to, thereby promoting the interpretation of the Experienced as Source » (Talmy 2000 : 116). En tout état de cause, certaines définitions de 'mots en $b l$-' évoquent la matérialisation d'un rayon qui relierait l'œil à l'objet, et dont la rupture fictive entraînerait la perte de la vision. Citons par exemple le sens $\mathrm{n}^{\circ} 6$ de blink $\mathrm{n}$. " an obscuration between the eye and an object » $(E D D)$; et le premier sens de blind n. : " anything which obstructs the sight or light $\gg(O E D)$.

Selon Talmy, le choix entre les deux possibilités répond à un principe cognitif global qui détermine quelle est la source du rayonnement: "The object that is taken to be the more active or determinative of the two is conceptualized as the Source of the Emanation » (ibid. : 117). Cependant, en ce qui concerne le mouvement fictif attribuée à la sensation ('Sensation paths'), et en particulier la vue, c'est en général l'observateur qui est conçu comme source de la perception: "This is the case for English, where some forms with the Experienced as Source offer difficulty to some speakers, and the use of a verb like show is minimal relative to that of a verb like see. Further, agentive verbs of vision in English are exclusively lexicalized for the Experiencer as subject and can take directional phrases only with the Experiencer as Source $^{29} »$ (ibid.: 116). Les propositions de Talmy s'avèrent toutefois utiles pour notre propos lorsqu'il s'agit de rendre compte de la polysémie des quatre verbes blush, blenk, blink $(O E D)$, et blinter $(E D D)$, déjà mentionnés dans notre analyse sémantique. En effet, ces verbes, qui signifient à la fois 'to cast a glance' et 'to shine', s'inscrivent parfaitement dans le cadre du double sens attribué au déplacement fictif postulé pour la perception visuelle.

\subsection{4. «Ghost Physics ${ }^{30} »$}

Selon Talmy, le type de conceptualisation exposé dans les paragraphes précédents, où une situation statique est perçue et conçue ${ }^{31}$ en termes de mouvement, englobe également des phénomènes culturels : certaines formes d'iconographie populaire (voir ci-dessus), ainsi que les croyances en des forces occultes, relèveraient du même processus : " It thus seems that the general fictivity complex generates the imaginal schemas of fictive motion not only in the cognitive systems of language and of visual perception, but also in that of cultural cognition, specifically in its conceptualizations of spirit and power. That is, in the cognitive culture system, the structure of such conceptions as ghost phenomena, harmful influence, and magical energy appears not to be arbitrary » (Talmy $2000: 128$ ).

Or, comme nous l'avons déjà noté dans le paragraphe consacré aux réseaux conceptuels métaphoriques (voir 7.1.2), une sous-classe non négligeable de 'mots en $b l$-' fait référence à des faits qui échappent à l'explication rationnelle, et dont l'origine mystérieuse est attribuée par la croyance populaire à des puissances occultes. Nous avons cité plus haut les définitions (parfois polysémiques) de blas (EDD), blast, blight et blink; ajoutons à présent celle de 1'OED pour blasted, très explicite quant au processus cognitif supposé : «Balefully or

\footnotetext{
${ }^{28}$ Talmy propose les énoncés parallèles : Even a casual passerby can see the old wallpaper through the paint / The old wallpaper shows through the paint even to a casual passerby. Cette double direction possible de la sensation nous rappelle les deux théories antiques de la vision, celle de l'émission et celle de l'émanation.

${ }^{29}$ Talmy propose l'exemple suivant : I looked into/toward/past/away from the valley (Talmy $2000: 116$ ).

${ }^{30}$ C'est ainsi que l'anthropologue Pascal Boyer nomme l'ensemble des propriétés particulières attribuées aux esprits et aux fantômes dans les systèmes de croyances de nombreuses traditions culturelles. (Talmy 2000 : 126).

${ }^{31}$ Afin d'éviter la distinction entre perception et conception, et de prendre en compte l'existence d'un continuum reliant les divers processus cognitifs qui entrent en jeu, Talmy suggère d'utiliser le terme de 'ception' : « While perhaps best limited to the phenomena of current processing, ception would include the processing of sensory stimulation, mental imagery, and currently experienced thought and affect » (Talmy $2000: 139-140$ ).
} 
perniciously blown or breathed upon; stricken by meteoric or supernatural agency, as parching wind, lightning, an alleged malignant planet, the wrath and curse of heaven ». Le second sens de bless témoigne également de l'existence de ces croyances en des puissances maléfiques : " to sanctify by making the sign of the cross ; usually as a defence against evil agencies » $(O E D)$. Le dictionnaire fait même explicitement état de tels systèmes de croyances dans les origines du mot: "The streaking of the lintel and doorposts with blood, to mark them as holy to the Lord and inviolable by the destroying angel, was apparently the kind of idea expressed by blóedsian in pre-christian times » (OED : 281). Ainsi, si l'étymologie ${ }^{32} \mathrm{de}$ bless établit effectivement la référence au sang, à partir de l'idée de sacrifice et de sang versé ; l'utilisation de la notion de Fictive Motion pourrait constituer un argument supplémentaire permettant d'étayer l'appartenance de ce mot à la classe des 'mots en bl-' renvoyant à la notion de $<$ Flot/Courant $>$, cette fois-ci sur des bases cognitives et culturelles.

Pour conclure, nous avons proposé plus haut $(\S 5.1 .2)$ l'hypothèse d'une projection conceptuelle métaphorique entre le domaine des phénomènes météorologiques et celui des croyances populaires, sur la base d'inférences opérées à partir de l'action de l'air et du vent. Le concept de Fictive Motion complète ce tableau et permet d'envisager certains référents comme correspondant à la conceptualisation de forces mystérieuses sous la forme d'un mouvement invisible ${ }^{33}$, dont le résultat tangible, probablement à l'origine de telles croyances, est d'ailleurs parfois désigné par un 'mot en $b l$-'. Citons par exemple blight : "Any malignant influence of obscure or mysterious origin; which withers hopes or prospects » $(O E D)$.

\subsection{Notion d'« Active Zone»}

Nous avons signalé plus haut dans notre analyse sémantique que certaines parties du corps apparaissent de façon récurrente dans les définitions: elles interviennent de façon systématique dans les procès désignés par certains 'verbes en $b l-$ '. Mais ces parties du corps, essentiellement la bouche et les yeux, ne sont pas toujours mentionnées de façon explicite. Par exemple, si les sens 1 et 3 de blabber dans l'OED font respectivement état de " to make sounds with the lips and tongue as an infant » et «Obs. To move the tongue between the lips in mockery », le sens 2 se limite à « to chatter, talk idly or senselessly ». De façon similaire, il est fait mention des yeux dans le sens 3 de blenk «Obs. To turn aside, raise (the eyes, eyebrows) » $(O E D)$, mais pas dans le sens 7 , à savoir « to cast a glance, to give a look ». En discours, ces verbes ont pour sujet un nom ou un pronom représentant un individu tout entier, alors que l'agent du procès, au sens strict, n'est qu'une partie du corps de cet individu. Pour l'énoncé David blinked, par exemple, Langacker commente : «The subject names a person, but the activity of blinking is not something carried out by a whole person - only the eyelid really does anything » (Langacker $2002: 189$ ).

\footnotetext{
32 « The etymological meaning was thus 'to mark (or affect in some way) with blood (or sacrifice); to consecrate' " $(O E D:$ 281). Onions atteste de même : "A purely English formation. OE. blētsian, blēdsian, bloedsian < *blöðisōjan, formed on blōðam 'blood'; the etymol. meaning being 'mark so as to hallow with blood'»(Onions 1966 : 100).

${ }^{33}$ Parmi les formes d'imagerie populaire représentant figurativement ce mouvement, Talmy cite les exemples des poignards assassins que lancent les yeux des personnages de bandes dessinées, et les rayons destructeurs qui semblent prolonger les doigts de certains sorciers. Il n'envisage pas la représentation de souffles délétères émis par la bouche des personnages (Talmy 2000 : 125).
} 


\subsubsection{Approche de Langacker}

Langacker propose d'explorer ce phénomène en introduisant la notion d' "Active Zone ». Dans des énoncés de ce type, le procès désigné par le verbe ou l'adjectif (ce que Langacker nomme 'relational predication') ne fait intervenir qu'une partie de l'objet repéré (trajector) et/ou du repère (landmark): "Those portions of a trajector or landmark that participate directly in a given relation will be referred to as its " active zone " with respect to the relation in question » (Langacker 2002: 190). Dans la plupart des relations prédicatives, Langacker observe systématiquement un écart entre l'entité profilée par une expression ou un lexème et son "active zone » : "In fact, a bit of reflection reveals that a discrepancy between profile and active zone represents the NORMAL situation [...] we find that only selected facets of a designated individual participate in the process, and that these facets differ from one expression to the next. [...] Some discrepancy between active zone and profile is thus the rule, not the exception » (ibid. : 191). Cet écart est d'ailleurs nécessaire à la souplesse du système linguistique : si, dans une relation prédicative, les entités profilées (trajector et/ou landmark) et leur 'active zone' devaient parfaitement coïncider, il y aurait alors prolifération de locutions alambiquées dont l'extrême précision ferait écran à la compréhension. Langacker donne quelques échantillons des énoncés que l'on pourrait envisager si tel était le cas: Roger's eyelids blinked, mais aussi The color sensation associated with the outer surface of this pen, the color sensation associated with the marks created by which is red, is yellow (This red pen is yellow). (ibid. : 192-193).

\subsubsection{Zone focale}

Langacker ajoute deux précisions concernant l' «Active Zone»: la région du corps concernée est floue, non strictement délimitée, et variable selon les occurrences, certaines sous-parties participant de façons diverses au procès : "The active zone should not be thought of as a discrete or sharply bounded region within the overall entity - it is more accurate to think of it as the focal area of the relational interaction » (Langacker 2002 : 190).

En ce qui concerne la sous-classe des 'verbes en bl-' renvoyant à la notion $<$ Flot/Courant $>$, tels que, entre autres, blab, blabber, blathe, blare ou bleat, c'est toute la région buccale au sens large qui est concernée, comprenant les lèvres, la langue, les mâchoires, les muscles articulatoires et orbiculaires, le larynx, les cordes vocales, et plus généralement l'ensemble de l'appareil vocal. Pour blare «roar with prolonged sound in weeping ", l' " active zone » s'étend à d'autres muscles du visage et aux yeux. Enfin, la notion d' «active zone» appliquée à l'activité désignée par blubber "to sob unrestrainedly or noisily » permet de jeter un pont entre les deux notions examinées ici, à savoir $<$ Flot/Courant $>$ et $<$ Vision/Luminosité $>$, la région buccale et les yeux paraissant prendre une part égale dans les procès désignés.

Si nous considérons à présent la sous-classe des 'verbes en $b l$-' renvoyant à $<$ Vision / Luminosité>, le caractère imprécis et composite de l' "active zone » concernée nous permet de dépasser de beaucoup le globe oculaire (blaze), et d'inclure au moins les paupières (blink), les cils, les muscles oculaires (blench: "Obs. to turn the eyes aside or away »), les orbites, ainsi que les canaux lacrymaux (blear Obs.), les nerfs optiques et même les sourcils (blenk). A sa manière, le lexicographe indique d'ailleurs pour certains de ces verbes l'impossibilité de délimiter quelle facette de l' « active zone » est profilée dans tel ou tel emploi. Pour blink, par exemple, l'OED précise : " Used in various shades of meaning which run into one another; in the earlier, the notion of 'glancing' predominates, in the later, that of 'winking' ». Quant à 
blind v., si le premier sens a trait à la perception visuelle ( " to make blind, deprive of sight » $O E D)$, le second fait explicitement référence aux mouvements des paupières : "To close the eyes of the understanding or moral perception; to deceive, 'throw dust in the eyes' » $(O E D)$.

\subsubsection{Association par référence à une pratique conventionnelle}

L' «active zone » peut recouvrir beaucoup plus qu'une sous-partie d'une entité ou une région contiguë, elle se définit par rapport à la fonction ou à l'activité caractéristique de celleci : «A second exacerbating factor is that the active zone is often not even a subpart of the entity designated by a nominal expression. Frequently it is something merely associated with the designated element in some characteristic fashion » (Langacker 2002 : 192). Par exemple, l'énoncé She heard the piano sera systématiquement compris comme signifiant She heard the sound of the piano, l'objet physique s'effaçant derrière l'instrument de musique. Nous avons déjà mentionné les divers procès qui s'apparentent aux sanglots (ajoutons blirt $(E D D)$ «Dial. to cry, weep, shed tears »), les mouvements des yeux, des paupières, des muscles oculaires (blunk $(E D D)$ " to scowl, frown ») : la notion d' " active zone » permet de rendre compte des sens de ces 'verbes en $b l$-' pouvant apparaître de prime abord bien éloignés de l'exercice de la vue au sens strict.

\subsubsection{Blame et blaspheme}

Pour ce qui est de la notion <Flot/Courant>, si nous considérons que l' " active zone » correspondant aux procès désignés par les 'verbes en $b l$-' concernés est constituée de la région buccale dans son ensemble et des fonctions afférentes, il nous semble possible d'envisager blame et blaspheme comme autant de manifestations de la relation prédicative profilant le même type de relations. En effet, si le premier sens de blame en anglais moderne (OED) est « to find fault with », le second, obsolète, paraît confirmer notre hypothèse : il s'agit de " to address with rebuke; to scold», pour lequel les réprimandes sont présentées, plus concrètement, comme issues de la bouche pour atteindre un destinataire.

Quant à blaspheme, «to utter profane or impious words", il met en jeu la même "active zone » : la bouche. Nous pouvons ici faire un rapprochement avec le sàr, l'une des langues du Tchad. Le sàr conceptualise en effet certaines 'paroles solennelles' à partir de la région corporelle émettrice. Une fois de plus, la notion d' " active zone » s'avère très utile : « [...] il existe, à côté de la parole ordinaire, une parole exceptionnelle, solennelle (qui rejoint, à certains égards, ce que Benveniste appelle la parole «performatrice »). En ce qui intéresse directement notre propos, c'est que pour cette parole qui engage plus étroitement celui qui la prononce, nous trouvons des expressions avec des noms de parties du corps [bouche, gorge, voix] » (Fédry 1976 : 100). Ainsi, l'acte de proférer une parole solennelle de bénédiction ou de malédiction sera rendu par l'équivalent de 'j'extrais ma bouche', 'maudire' par 'jeter la voix', et ‘je promets' sera ‘je jette ma gorge' (ibid. : 99-100).

\subsection{5. « Active Zone » et étymologie}

Si les traits définitoires n'y font allusion que de très loin, l'étymologie mentionne parfois explicitement l' " active zone », comme c'est le cas pour blet : « [<F. blet-ir 'devenir blet', f. blet 'sleepy' as an over-ripe pear]. To become 'sleepy', a form of decay to which fleshy fruits 
are subject » $(O E D)$. Une fois de plus, il est fait référence, à l'origine, au mouvement caractéristique des paupières, qui se ferment lorsque survient le sommeil. Pour blunt, de même, la notion d' «active zone» permet d'inclure comme appartenant à la sous-classe considérée ce lexème, dont les sens actuels, à savoir, « 1. dull, insensitive, stupid, obtuse ; 2. of an angle, edge or point : not sharp, obtuse », semblent au premier abord très éloignés de ce dont il est question dans ces lignes. L'OED précise en effet: «[Etymology unknown: perhaps some connexion with ON. blunda to doze, blunda augum to shut the eyes, blundr dozing, sleep]». De plus, le sens noté 1 s'appliquait originellement aux yeux : "said originally of the eyes, whence of the perceptions, and the intellect ». Quant à blear v., il est attesté dans l'OED sous deux entrées différentes, notées $\mathrm{v}^{1}$ et $\mathrm{v}^{2}$. L' « active zone » de $\mathrm{v}^{1}$ « to $\operatorname{dim}$ (the eyes) with tears or inflammation » correspond aux yeux; alors que celle de $\mathrm{v}^{2}$ (sens obsolète) est la région buccale : " to protrude the tongue in mockery ${ }^{34}$ ». Ici, la notion d'« active zone » permet d'éclairer les remarques préliminaires du lexicographe: d'une part, nous avons pour $\mathrm{v}^{2}:$ : [apparently distinct from the prec. : perhaps onomatopœic, but naming a gesture rather than a sound, though some inarticulate sound may have originally been implied] ».

D'autre part, si nous prenons en compte l'origine de blear adjectif, dont le verbe est selon l'OED fort probablement issu, le sens de ce mot, à savoir «ME. blere, an epithet of the eyes » s'avère particulièrement vague, mais renseigne de façon très lâche sur l' " active zone » de blear $\mathrm{v}^{1}$. En outre, l'OED rejette comme « peu probable » le lien qui apparenterait blear (adj.) au suédois plira et au danois plire, 'to blink, leer' ; mais mentionne néanmoins ces deux mots, dont le sens désigne, comme blear $\mathrm{v}^{2}$, un mouvement. Ces remarques lexicographiques,

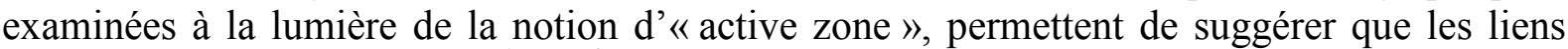
qu'entretiennent les sens de $\mathrm{v}^{1}$ et $\mathrm{v}^{2}$ proviennent, entre autres, de la référence commune à un mouvement de deux parties du corps, celles correspondant aux deux sous-classes considérées ici.

En conclusion, la notion d' " active zone » proposée par Langacker, apporte une cohérence supplémentaire à notre analyse et à la constitution des deux sous-classes proposées : les 'verbes en $b l$-' dont les sens renvoient à la notion <Flot/Courant $>$ s'articulent autour de la région buccale au sens large; et l'on peut envisager que la zone oculaire constitue le point d'ancrage de ceux renvoyant à la notion $<$ Vision/Luminosité $>$.

\subsection{Polysémie de blood : « Extraction de schemas »}

\subsubsection{Modèle en réseau ('network model')}

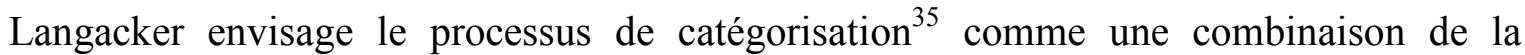
catégorisation par prototype et de la catégorisation à partir de schemas, abstractions que l'esprit effectue à partir de points communs repérés dans diverses actualisations du 'pôle

\footnotetext{
${ }^{34}$ Une des citations fournies par l'OED étant, par ex. (1616) : «All that the silly sheep can do, is only to bleare and bleat a little with his tongue » $(O E D: 274)$.

${ }^{35}$ Nous rappelons les définitions que donne Langacker des deux types de catégorisation reconnus par la plupart des tendances de la linguistique cognitive : «A prototype is a typical instance of a category, and other elements are assimilated to the category on the basis of their perceived resemblance to the prototype ; there are degrees of membership based on degree of similarity. A schema, by contrast, is an abstract characterization that is fully compatible with all the members of the category it defines (so membership is not a matter of degree) ; it is an integrated structure that embodies the commonality of its members, which are conceptions of greater specificity and detail that elaborate the schema in contrasting ways » (Langacker 1987 : 371).
} 
sémantique' d'un lexème : "My own proposal, the network model, represents a synthesis of prototype theory and categorization based on schemas » (Langacker 2002 : 266).

La structuration globale d'une catégorie s'effectue à partir du prototype, à savoir l'élément qui, situé au "centre de gravité » du réseau, apparaît comme le plus naturellement saillant, celui auquel le locuteur fait le plus souvent référence, et le plus susceptible d'être choisi comme représentant d'une catégorie donnée (Langacker 1987 : 492). Pour blood, le prototype est vraisemblablement représenté par le sens classé en première position par l'OED sous l'entrée blood, à savoir : "The red liquid circulating in the arteries and veins of man and the higher animals, by which the tissues are constantly nourished and renewed $\gg(O E D: 302)$. Ce prototype occupe la place centrale du réseau; d'autres membres de la catégorie blood, bien que présentant des disparités plus ou moins importantes, seront néanmoins assimilés à celle-ci comme membres périphériques, à des distances variables, s'ils sont jugés suffisamment semblables au prototype. Le sens obsolète « rake, roisterer », par exemple, est plus éloigné du prototype que ne l'est « the corresponding liquid, coloured or colourless, in animals of lower organization $»$.

\subsubsection{Extension du réseau}

L'appartenance à une catégorie est donc une question de degré; et le réseau peut s'enrichir de nouveaux membres selon la diversité des contextualisations et la sanction de la convention. La structure se construit le long de deux axes : elle se développe à la fois "par le haut' (extraction d'un schema plus abstrait qui en subsume deux ou plusieurs de rang inférieur dans la hiérarchie), et 'par le bas' par extension (spécialisation) à partir du prototype. Le nombre et la variété de ces opérations ne sont pas prévisibles : «We cannot be certain how far 'upward' a speaker extends this network through the process of abstraction (schematization), and, in particular, whether he extracts a 'superschema' having all the other nodes as direct or indirect instantiations. Nor do we know how far 'downward' a speaker articulates the network into progressively more specialized notions » (Langacker 2002 : 267).

Ainsi, lorsque blood, à partir de son sens prototypique, désigne le même type de liquide vital, circulant non plus chez l'homme et les animaux supérieurs, mais dans l'organisme d'animaux inférieurs, et que sa couleur n'est plus un critère d'appartenance à la catégorie (cf. " coloured or colourless »), il se produit conjointement l'extraction d'un schema de rang supérieur, que nous pourrions nommer [BLOOD'], nous inspirant de Langacker pour [TREE] (Langacker 1987 : 381). Il est difficile d'appréhender l'ensemble des connaissances liées à un lexème donné, actualisées en contexte par un locuteur donné sous la forme des différents sens que peut prendre celui-ci; mais il est vraisemblable que [BLOOD'], superordonné à [BLOOD], comporte une spécification de moins, celle liée à la couleur rouge. [BLOOD'] pourrait correspondre à l'étiquette schématique : [Vital liquid circulating in animate beings].

De même, l'extension permettant de recouvrir le sens «Applied to liquids or juices in some way resembling or suggesting [human blood], esp. to the sap of plants ", donne lieu à l'extraction du schema [BLOOD']. Nous proposons d'expliciter sommairement [BLOOD'] par la formule : [Vital liquid circulating in living organisms]; un processus de généralisation et d'abstraction permettant de désigner par blood un liquide incolore circulant dans des organismes inanimés. [BLOOD], [BLOOD'], et [BLOOD''] sont autant de points d'entrée ('access nodes') permettant d'accéder à l'ensemble du réseau conceptuel désigné par blood.

Enfin, une dernière extraction de schema, [BLOOD','], hyperordonné aux trois précédents, permet l'extension de sens : «Blood shed; hence bloodshed; manslaughter, death », dont l'explicitation succincte serait: [Vital liquid normally circulating in living 
organisms, which, if shed, may bring about death]. Il apparaît que [BLOOD','] inclut les deux modes complémentaires de représentation du sang, que nous avons déjà évoqués, à savoir sang intérieur et sang extérieur, matérialisés dans certaines langues issues de l'indo-européen, dont le latin, par deux mots différents (cf. Lat. sanguis et cruor).

\subsubsection{Autres domaines, autres sens de blood}

Il ne semble pas possible de conjecturer l'extraction d'un schema hyperordonné à l'ensemble pour l'extension : "The supposed seat of emotion, passion; as in some expr. 'it stirs the blood', 'it makes the blood creep' etc. ». En effet, la configuration des catégories selon Langacker ne présuppose pas l'existence d'une 'superschema' qui serait compatible avec tous les autres membres de la catégorie : "Extensions can occur without the motivating schemas (similarity-perceptions) achieving the status of conventional units. Thus a network need not incorporate a well-behaved schematic or taxonomic hierarchy, with a single topmost node that dominates all the others » (Langacker 1987 : 381). Selon l'approche de Langacker, les catégories peuvent en effet être élargies par d'autres principes cognitifs généraux, tels que métaphore, métonymie, ou transformation de schèmes ${ }^{36}$ (Cuyckens \& Zawada 2001 : intr. xiv). En outre, la plupart des items lexicaux ne s'appréhendent que par rapport à plusieurs domaines d'expérience (Langacker 2002 : 5). Le sens noté plus haut correspond au résultat d'un transfert conceptuel opérant entre le domaine source du 'Corps Humain' et le domaine cible des 'Etats et Affects'. Les inférences que l'esprit établit à partir des propriétés du sang, en particulier sa couleur, sa température, la vitalité dont il fait preuve en abreuvant et nourrissant les tissus ${ }^{37}$, permettent de conceptualiser et de nommer certains états affectifs par le même lexème.

Enfin, le sens « race and kindred as connoted by blood» semble être accessible par le même processus de transfert métaphorique, cette fois-ci entre le domaine du 'Corps Humain' et celui des 'Relations Familiales et Humaines'. Les inférences conjecturées par Lakoff et Johnson dans ce type de processus cognitif ne semblent pas émaner des propriétés aspectuelles et fonctionnelles du sang, mais pourraient s'appuyer sur le rôle que joue celui-ci dans la survie de l'organisme, ou plutôt de l'être tout entier. Les propriétés attribuées au sang proviennent d'ailleurs souvent de conceptions anciennes erronées, comme le mentionne l'OED pour l'ensemble des sens qu'il regroupe dans sa seconde rubrique : "[states of the body] often derived from earlier superficial or erroneous notions of its character and action ».

\subsection{Schème mental <CONTAINER $>$}

\subsection{1. 'Schèmes mentaux' de Johnson}

« Image schemas are imaginative, non-propositional, and dynamic patterns of our bodily movements through space, manipulations of objects, and perceptual interactions. They have an internal structure and can be metaphorically elaborated to provide for our understanding of abstract concepts » (Soares da Silva, 2003 : 282). Les 'schèmes mentaux' de Johnson sont donc des représentations conceptuelles qui émergent à partir de l'expérience sensori-motrice. Ils opèrent à un niveau d'abstraction et de généralité qui se situe en aval des images mentales

\footnotetext{
${ }^{36}$ Transformation de schèmes : au sens où l'entendent Lakoff et Johnson, par ex. Johnson 1987 : 104.

${ }^{37} \mathrm{Cf}$ la conception platonicienne : « [Les dieux] ont creusé à travers notre corps des canaux, pareils aux canaux qu'on creuse dans les jardins, afin qu'il fût irrigué comme un jardin par l'eau qui y coule » (Timée : 195).
} 
concrètes directement issues de la perception et de l'action, et en amont des structures propositionnelles abstraites : (ibid. : 29). Ils sont à la base de l'élaboration de la représentation de l'expérience, et constituent la première étape de la distanciation vis-à-vis de l'événement concret, afin d'évoluer peu à peu vers l'abstraction. Ce mouvement n'est d'ailleurs pas sans rappeler, en phylogénèse, le passage de la culture épisodique à la culture mimétique, lorsque l'esprit acquiert les capacités de modélisation des événements et de différenciation de la référence, et visant à une communication volontaire ${ }^{38}$.

Les schèmes fondamentaux, ceux qui donnent le plus fréquemment cohérence et organisation à l'expérience, et interviennent de façon récurrente dans la structuration de la pensée, sont au nombre d'une vingtaine. Gibbs et Colston 1995 en citent huit : CONTAINER, BALANCE, SOURCE-PATH-GOAL, PATH, CYCLE, ATTRACTION, CENTERPERIPHERY, et LINK. Le premier exemple que donne Johnson est celui de VERTICALITY : "We grasp this structure of verticality repeatedly in thousands of perceptions and activities we experience every day, such as perceiving a tree, our felt sense of standing upright, the activity of climbing stairs [...]» (Johnson 1987 : xiv).

\subsubsection{Le schème $<$ CONTAINER $>$}

Selon Lakoff, le schème CONTAINER présente une structure très simple composée de trois éléments interdépendants organisés en gestalt: intérieur, extérieur, et frontière. Il provient, d'une part, de la conceptualisation du corps en tant que contenant, basée sur les fonctions vitales d'ingestion, d'excrétion, et de respiration; d'autre part de l'interaction du corps avec le milieu, où le corps est conçu comme un objet se situant soit à l'intérieur d'un contenant, soit hors de celui-ci : "The CONTAINER schema defines the most basic distinction between IN and OUT. We understand our own bodies as containers -- perhaps the most basic things we do are ingest and excrete, take air into our lungs and breathe it out. But our understanding of our own bodies as containers seems small compared with all the daily experiences we understand in CONTAINER terms ${ }^{39}{ }$ (Lakoff $1987: 271$ ).

La définition que propose Johnson est très voisine, en ce qu'elle souligne le caractère omniprésent et fondamental de ce schème, ainsi que la place centrale du corps dans sa structuration interne : "Our encounter with containment and boundedness is one of the most pervasive features of our bodily experience. We are intimately aware of our bodies as threedimensional containers into which we put certain things (food, water, air) and out of which other things emerge (food and water wastes, air, blood, etc.). From the beginning, we experience constant physical containment in our surroundings (those things that envelop us). We move in and out of rooms, clothes, vehicles, and numerous kinds of bounded spaces. We manipulate objects, placing them in containers (cups, boxes, cans, bags, etc.) » (Johnson $1987: 21)$.

A partir du schème <CONTAINER $>$ s'élabore l'orientation intérieur-extérieur, concernant d'abord un espace borné à trois dimensions, puis appliquée à des domaines à deux, ou à une seule dimension. Les inférences liées à ce schème incluent les notions de résistance, ou de protection, par rapport à des forces externes ; ainsi que la limitation ou la restriction des

\footnotetext{
${ }^{38}$ Donald (1991 : 213) : «Le plus haut niveau de traitement dans le cerveau entraîné au mimétisme n'est plus l'analyse et la décomposition des événements perceptifs mais la modélisation de ces événements dans des actes moteurs auto-initiés».

39 Suit une longue citation de Johnson (1987: 30-31), décrivant quelques-unes des activités matinales quotidiennes désignées par des expressions contenant in/out, telles que 'wake out of sleep', 'walk in a daze out of your bedroom', 'look in the mirror', 'take out the toothpaste', etc.
} 
forces internes. De plus, l'acte de circonscrire un domaine donné impose une certaine fixité à la localisation, et la possibilité d'être accessible on non à l'observateur (ibid. : 21-22). La structuration de ce schème et les inférences qui en découlent imposent des contraintes sur les possibilités de projection métaphorique, et nous permettent de concevoir de nombreux domaines abstraits en termes de <CONTAINER > : les catégories au sens large (Lakoff 1987 : 300), les catégories lexicales et syntaxiques, les ensembles mathématiques, mais aussi les états et les activités, ou la structure argumentative ; et enfin, les émotions (cf. la célèbre étude de la colère (anger) dans Lakoff 1987 : 380-416, et Kövecses ${ }^{40}$ 2000). Enfin, l'activité linguistique peut également s'appréhender par l'intermédiaire de ce schème : la bouche est conçue comme le contenant d'où s'échappent les paroles : « [...] such that when the container is open, linguistic action is possible, and when it is closed, there is only silence » (Gibbs $2006: 104)$.

La conceptualisation de la parole présentée ici n'est d'ailleurs que l'une des multiples manifestations de la "métaphore du conduit » (conduit metaphor), dont Reddy 1993 a été le premier à décrire l'omniprésence en anglais, et, plus généralement, dans la culture occidentale. Des centaines d'expressions sont imputables à cette métaphore conceptuelle qui conduit à envisager les mots comme autant de réceptacles tangibles contenant une substance, le sens, lui-même dépositaire de la pensée, pensée ainsi véhiculée ${ }^{41}$ de la bouche du locuteur (sortie du corps) à l'oreille de l'interlocuteur (entrée dans un autre corps). Bien que nous puissions, avec Reddy et d'autres auteurs (comme Lakoff \& Johnson 2003 : 12; 206), déplorer le caractère 'pernicieux ${ }^{42}$ de la métaphore du conduit, il n'en demeure pas moins que les sens d'un nombre substantiel de 'verbes en $b l$-' semblent renvoyer à une conceptualisation de la communication langagière dans laquelle l'assise principale serait constituée par le schème $<$ CONTAINER $>$. Nous en voulons pour preuve par exemple les sens de blab dans des citations fournies par l'OED: "The Chamber of the House of Commons should be fitted with 'blab-off switches'... These 'popular devices enabled American viewers (of television) to switch off advertisers' annoucements' "; "Others...ignore the commercial interference...without benefit of blab-off devices ». Les paroles importunes sont envisagées comme un flot de liquide dont on pourrait arrêter le cours grâce à un dispositif s'apparentant à un robinet.

\subsubsection{Origine corporelle du schème $<$ CONTAINER $>$ : témoignage du sàr}

Dans ses études de la langue sàr (Tchad), Fédry a pour objet essentiel de montrer que l'orientation spatiale, entre autres, puise ses origines dans la représentation du corps et de ses dimensions cardinales : "C C'est sur le 'patron' de cette première expérience de mon corps se situant dans le monde, et situant toute chose par rapport à lui, que se construisent l'orientation et la situation réciproque des objets » (Fédry 1976 : 75). La plupart des morphèmes désignant les relations d'orientation, de localisation, de mouvement, et même diverses relations logiques, sont initialement des noms de parties du corps : "C'est que le corps apparaît ici

\footnotetext{
${ }^{40}$ La projection conceptuelle est décrite par la métaphore 'Anger Is a Hot Fluid In a Container', où le corps humain est conçu comme un 'contenant' clos soumis à diverses forces internes et externes, et à l'influence de la chaleur (Kövecses $2000: 20-35 ; 161$ ).

${ }^{41}$ Voir par ex., chez Homère et Hésiode : les «paroles ailées » qui s'échappent de l'espace clos du corps et quittent «l'enclos [des] dents » (Odyssée : p. 855).

${ }^{42}$ L'un des effets pervers de la métaphore du conduit est d'induire une réification du sens, qui fige ce dernier une fois pour toutes et ne permet pas de se placer dans la perspective de la 'construction du sens' : «Meanings are objects, with fixed and determinate properties, assigned to linguistic expressions » (Cuyckens et al. 2003: 12).
} 
comme un symbole organisateur, un principe structurant de la langue, auquel peu de zones du champ sémantique échappent » (ibid. : 100-102).

La première composante du schème $<$ CONTAINER $>$, la notion d'intérieur, est rendue en sàr par deux termes différents, selon que les limites de l'objet repéré sont bien définies ou, au contraire, vagues : «La langue sàr distingue deux relations d'intériorité, exprimées par kèm'ventre' (objet à contour délimité) et kùm- 'œil' (espace non fermé par une limite [...]), marquées par un morphème spatio-temporel» (ibid. : 78). Fédry formule deux explications permettant de rendre compte du sens insolite de 'dans' pour le terme désignant l'œil. D'une part, «l'expérience du regard comme réceptivité illimitée, qui 'enveloppe', sans enfermer dans un contenant délimité, aurait ainsi fourni le modèle de la notion de 'milieu ambiant' »; d'autre part, «l'expérience du regard pénétrant l'eau ; l'expérience de l'eau serait première et fournirait le modèle de tout milieu ambiant. [...] La masse translucide de l'eau, c'est donc son 'œil' » (ibid.: 79). En revanche, la seconde dimension de <CONTAINER $>$, la notion d'extérieur, n'est pas rendue par un mot désignant une partie du corps : « Le terme exprimant la notion de 'dehors' n'est pas un terme du corps. On le comprend; si le 'ventre' fournit un symbole spontané de l'intériorité, aucune partie du corps ne saurait signifier l'extériorité » (ibid. : 80).

Par ailleurs, le regard peut également être conceptualisé comme un mouvement ${ }^{43}$, ainsi rendu à partir de l'idée de sortir, et surtout de se répandre au-dehors, d'embrasser une partie de l'espace. C'est le même mot kùm 'œil' qui, combiné à d'autres, est utilisé pour exprimer le déplacement dans l'espace : kùm s'est transformé en une préposition marquant d'abord une localisation statique, puis une localisation dynamique (Fédry 1976 : 82). Le phénomène de grammaticalisation $^{44}$ affectant kùm atteste une conceptualisation particulière de la vue : celleci est envisagée comme un rayonnement s'écoulant du corps (le 'container'), par les yeux, ouvertures ménagées dans la frontière avec l'extérieur, frontière que constitue la peau.

Si le schème $<$ CONTAINER $>$ a bien une réalité psychologique, il semblerait que la langue sàr fournisse ici un témoignage permettant d'envisager son origine comme issue de l'expérience corporelle, et corroborant en cela les idées de Johnson, que nous rappelons : " Our encounter with containment and boundedness is one of the most pervasive features of our bodily experience » (Johnson 1987 : 21). C'est l'interaction directe du corps avec le milieu, que ce soit en tant que repère (landmark, ici le contenant), ou en tant qu'entité repérée (trajector, ici l'objet contenu dans un autre), qui est à l'origine de l'orientation intérieurextérieur et de l'émergence du schème $<$ CONTAINER $>$. Or, il semblerait que la conceptualisation des référents désignés par de nombreux 'mots en $b l$-' repose sur une base fournie principalement par la structuration de ce schème, où l'intérieur du corps est relié à l'extérieur par divers mouvements de flux et de reflux qui s'exercent dans des lieux d'entrée et de sortie situés sur la frontière constituée par la peau.

\subsection{4. 'Mots en $b l$-' et schème $<$ CONTAINER $>$}

En effet, en ce qui concerne les sens des lexèmes des deux sous-classes de 'mots en $b l$-' examinées ici, nous avons noté que les orifices du visage interviennent de façon directe ou indirecte dans les traits définitoires; la première sous-classe est ancrée autour des yeux, et la seconde autour de la bouche. Pour chacune de ces sous-classes, les sens des mots ont trait,

\footnotetext{
${ }^{43}$ Le sàr paraît témoigner de certaines conceptualisations rejoignant l'une des deux théories antiques de la vision, celle de «l'émission », où l'œil est conçu comme émettant des rayons visuels (Imbert 2005 : 91-93).

44 "Grammaticalization is defined as the development from lexical to grammatical forms and from grammatical to even more grammatical forms » (Heine \& Kuteva $2002: 2$ ).
} 
d'une part, à la fonction principale ou aux diverses activités de ces parties du corps, et, d'autre part, aux sécrétions qui s'écoulent de celles-ci. Ainsi pour la première, de nombreux termes désignent soit l'acte de voir ou de briller (blaze : " to shine brightly, glitter » $(O E D)$; blink: " cast or let fall a glance »), divers mouvements des yeux (blink), ou font référence aux pleurs (blirt : " an outburst of tears, a sudden fit of weeping » $O E D$ ). Les procès désignés par ces verbes peuvent s'envisager comme des écoulements de substances visibles (les larmes) ou invisibles (le 'rayon visuel') qui sortent du corps par les yeux.

En ce qui concerne la seconde sous-classe, la fonction désignée est principalement l'activité langagière (blab, blabber), ou plus exactement la production de sons et de bruits (bleat, blare). Les substances qui proviennent de la cavité buccale sont essentiellement de l'air (blow: "He blew a whiff from his pipe », citation de l'OED), mais aussi le flot de paroles, de chants, ou de cris qui s'en échappent pour atteindre l'oreille de l'interlocuteur ("Loud speakers blared across the water to us the eternal Turkish erotic whine »; "His voice blared through powerful amplifiers » citations $O E D)$. Ces sons peuvent être ceux émis par l'homme ou l'animal. Enfin, c'est la langue elle-même que l'on tire parfois hors de la bouche (blare, blate $(E D D)$, blear : "Writhing their mouths, blearing out their tongues », cit. $O E D)$.

Les nombreux cas de polysémie que nous avons recensés peuvent s'interpréter, au niveau cognitif, comme autant de traces des relations qu'entretiennent les deux notions : les divers sens des lexèmes renvoient à une même conceptualisation d'un 'mouvement sortant' du corps humain, que ce mouvement provienne de la bouche ou des yeux. La représentation mentale de ce 'mouvement sortant' pourrait émerger du schème fondamental $<$ CONTAINER $>$ tel que l'ont défini Lakoff et Johnson, et reposant sur les trois éléments interdépendants que sont l'intérieur, l'extérieur, et la frontière. Les inférences liées à ce schème, qui incluent les notions de résistance à des forces externes, ainsi que la limitation de forces internes, permettent de conceptualiser certaines interactions du corps avec le milieu sous la forme de mouvements entrant ou sortant. Ces mouvements transmis entre l'intérieur et l'extérieur, s'effectuent par des lieux de passage, où la frontière de la peau est interrompue, comme les orifices de la face.

Sur le plan linguistique, la caractéristique 'Paroxysme - Excès', relevée dans la plupart des traits définitoires, peut s'envisager comme un écho de la conceptualisation de cette dynamique des forces qui s'applique à la frontière entre intérieur et extérieur : les points de rupture sont étroits, les forces s'y engouffrent avec impétuosité. Nous avons vu que les mouvements sont en général des mouvements 'sortant', mais nous percevons le même trait saillant dans les sens de quatre verbes désignant des mouvements 'entrant' : blab, blabber, blybe, blyber (« to drink much and frequently/heavily/largely, to tope »EDD).

\section{Conclusions}

Ainsi donc, l'étude de ces deux sous-classes des 'mots en bl-' ayant un sens qui renvoie aux notions de 'Vision/Luminosité' et de 'Flot/Courant', qui représentent à elles deux les trois quarts de l'ensemble des 'mots en $b l$-', nous a-t-elle permis de dégager, au niveau sémantique, divers cas de régularité polysémique qui touchent un grand nombre de lexèmes. Ces cas de polysémie, ainsi que les traits saillants 'Paroxysme/Violence' et 'Désordre/Irrégularité', apparaissant de façon systématique dans les définitions, ont fait apparaître les liens qu'entretiennent les deux notions, et ont servi de point de départ à notre approche cognitive. 
Les phénomènes observés à la lumière d'une approche cognitive appelée «Embodiment», qui place le corps humain au centre des processus dont sont issues les fonctions cognitives, au moyen de projections métaphoriques reposant sur une structure sousjacente de 'schèmes mentaux', émergeant de l'interaction avec le milieu, ont conduit à la description de la façon dont l'esprit conceptualise et catégorise les référents désignés par les membres de ces deux sous-classes de 'mots en $b l$-' : il semblerait que les sens de ces derniers permettent l'accès à des représentations dans lesquelles le schème fondamental $<$ CONTAINER $>$ paraît jouer le rôle de soubassement initial.

Deux parties du corps, les yeux et la bouche, évoquées de façon explicite ou implicite dans les définitions, interviennent de diverses façons dans les représentations de procès et d'entités désignés par des 'mots en $b l$-'. Elles sont la plupart du temps conceptualisées comme les points de rupture pratiqués dans la frontière entre l'intérieur et l'extérieur du corps. Ces trois composantes structurent le schème $<$ CONTAINER $>$, selon lequel le corps est conçu comme un domaine clos dont la peau constitue l'enceinte. Dans ce cadre, les yeux et la bouche permettent la communication et les échanges entre intérieur et extérieur.

Nous avons évoqué les représentations que divers 'peuples anciens', et diverses philosophies antiques, avaient élaborées du corps humain, de ces deux parties du corps, et de leurs fonctions. Ces conceptualisations faisaient la part belle aux mouvements de flux et de reflux des éléments primordiaux, eau, air, feu, qui se produisent sans cesse entre macrocosme et microcosme. Les représentations auxquelles nous avons abouti au terme de cette étude constituent peut-être, par-delà le temps, un certain écho de ces conceptions-là.

\section{Références}

BARCELONA, Antonio 2003 [2000]. « On the plausibility of claiming a metonymic motivation for conceptual metaphor » in Metaphor and Metonymy at the Crossroads. A Cognitive Perspective, Antonio Barcelona (ed.). Berlin : Mouton de Gruyter, pp. 31-58.

BARCElona, Antonio (ed.) 2003 [2000]. Metaphor and Metonymy at the Crossroads. A Cognitive Perspective. Berlin : Mouton de Gruyter.

Buck, Carl D. 1949. A Dictionary of Selected Synonyms in the Principal Indo-European Languages. Chicago : University of Chicago Press.

Chantraine, Pierre 1999 [1968]. Dictionnaire étymologique de la langue grecque. Paris : Klincksieck.

CRYSTAL, David 2003. A Dictionary of Linguistics \& Phonetics (5 ${ }^{\text {ème }}$ éd.). Oxford: Blackwell.

Culioli, Antoine 1990. Pour une linguistique de l'énonciation, tome 1. Gap : Ophrys.

Cuyckens, Hubert, \& Britta ZawAda (ed.) 2001. Polysemy in Cognitive Linguistics. Amsterdam/Philadelphia : John Benjamins.

Cuyckens Hubert, René DiRven, \& John TAYlor (ed.) 2003. Cognitive Approaches to Lexical Semantics. Berlin/New York : Mouton de Gruyter.

DONALD, Merlin 1999 [1991]. Les origines de l'esprit moderne : trois étapes dans l'évolution de la culture et de la cognition. Traduction de la $1^{\text {ère }}$ éd. américaine par C. Emenegger et F. Eustache. Paris/Bruxelles : De Boeck University. 
DuMEZIL, Georges 1983. «Le sang dans les langues classiques », Nouvelle revue française d'hématologie vol. 25, pp. 401-404.

FEDRY, Jacques 1976. «L'expérience du corps comme structure du langage. Essai sur la langue sàr (Tchad) », L'Homme, XVI (1), pp. 65-107.

FIRTH, John R. 1930. Speech. London: Ernest Benn.

GÄRDENFORS, Peter 2000. Conceptual Spaces. The Geometry of Thought. Cambridge (Mass.) : MIT Press.

GiBBS, Raymond W. Jr. 2006. Embodiment and Cognitive Science. Cambridge : Cambridge University Press.

GIBBS, Raymond W. Jr. \& Herbert L. COLSTON 1995. « The cognitive psychological reality of image schemas and their transformations ", in Cognitive Linguistics 6-4, pp. 347-378.

HeINE, Bernd 1997. Cognitive Foundations of Grammar. New York: Oxford University Press.

HeINE, Bernd, and Tania Kuteva 2002. World Lexicon of Grammaticalization. Cambridge : Cambridge University Press.

Hesiode 1999. La Théogonie. Les Travaux et les Jours et autres poèmes. Trad. de P. Brunet et commentaires de M.C. Leclerc. Paris : Le Livre de Poche classique.

HOMERE 1955. Iliade-Odyssée. Paris : Gallimard (la Pléiade).

IMBERT, Michel 2005. «Le visible et la vue de l'Antiquité au Moyen-Âge», in La Lumière au siècle des Lumières et aujourd'hui, Jean-Pierre Changeux (dir.). Paris : Odile Jacob, pp. 90-102.

JoHnson, Mark 1987. The Body in the Mind: the Bodily Basis of Meaning, Imagination, and Reason. Chicago / London : University of Chicago Press.

JoUBAUD, Catherine 1991. Le corps humain dans la philosophie platonicienne. Paris: Librairie philosophique J. Vrin.

KöVECSES, Zoltan 2000. Metaphor and emotion: Language, culture, and body in human feeling. Cambridge, England : Cambridge University Press.

LAKOFF, George 1987. Women, Fire, and Dangerous Things. Chicago : University of Chicago Press.

LAKOFF, George, and Mark JoHnson 1999. Philosophy in the Flesh. The embodied mind and its challenge to Western thought. New York : Basic Books.

LAKOFF, George, and Mark JoHnson 2003 [1980]. Metaphors We Live By. Chicago: University of Chicago Press.

LANGaCKer, Ronald W. 1987. Foundations of Cognitive Grammar. Vol. I: Theoretical Prerequisites. Stanford, California : Stanford University Press.

LANGACKER, Ronald W. 2002 [1991]. Concept, Image, and Symbol. The Cognitive Basis of Grammar. (2 ${ }^{\text {ème }}$ éd.). Berlin / New York: Walter de Gruyter.

LAPAIRE, Jean-Rémi \& Wilfrid RoTGÉ 1998. Linguistique et grammaire de l'anglais. Toulouse : Presses Universitaires du Mirail.

Lincoln, Bruce 1986. Myth, Cosmos, and Society. Indo-European Themes of Creation and Destruction. Cambridge (Mass.) \& London : Harvard University Press.

LINKE, Uli 1985. «Blood as metaphor in Proto-Indo-European », in Journal of IndoEuropean Studies 13, pp. 333-376.

MCNeILL, David 2005. Gesture and Thought. Chicago : University of Chicago Press. 
MALlORY, James \& Douglas Q. ADAMs (eds.) 1997. Encyclopedia of Indo-European Culture. London \& Chicago : Fitzroy Dearborn.

OniANS, Richard B. 1999 [1951]. Les origines de la pensée européenne. Traduit de l'anglais par B. Cassin, A. Debru, M. Narcy. Paris : Le Seuil.

OnIOns, Charles T. (ed.) 1966. The Oxford Dictionary of English Etymology. Oxford: Clarendon Press.

Platon 1999 (4 $4^{\text {ème }}$ édition). Timée - Critias (Présentation et traduction par Luc Brisson). Paris : Flammarion.

RADDEN, Günter 2003 [2000]. «How metonymic are metaphors?» in Metaphor and Metonymy at the Crossroads. A Cognitive Perspective, Antonio Barcelona (ed.). Berlin : Mouton de Gruyter, pp. 93-108.

REDDY, Michael 1993 [1979]. "The conduit metaphor : A case of frame conflict in our talk about language ", in Andrew Ortony (ed.): Metaphor and Thought, $2^{\text {nd }}$ edition. Cambridge : Cambridge University Press, pp. 164-201.

SOARES DA SILVA, Augusto 2003. "Image schemas and category coherence : the case of the Portuguese verb deixar ", in Cognitive Approaches to Lexical Semantics, Cuyckens, Dirven, \& Taylor (ed.). Berlin/New York : Mouton de Gruyter, pp. 281-323.

SweEtSer, Eve 1990. From Etymology to Pragmatics. Cambridge/New York/Melbourne: Cambridge University Press.

TALmY, Leonard 2003 [2000]. Toward a Cognitive Semantics. Volume I: Concept Structuring Systems. Cambridge (Mass.) / London (England) : MIT Press.

TAYLOR, John R. 2003 (3rd ed.) [1989]. Linguistic Categorization. Oxford: Oxford University Press.

WatkIns, Calvert 2000 [1985]. The American Dictionary of Indo-European Roots. $2^{\text {ème }}$ édition, Boston : Houghton Mifflin.

Wright, Joseph (ed.) 1898-1905. The English Dialect Dictionary. Vols I-VI. London : H. Frowde. 